\title{
ARTICLE GITRL on inflammatory antigen presenting cells in the lung parenchyma provides signal 4 for T-cell accumulation and tissue-resident memory T-cell formation
}

\author{
Kuan-Lun Chu ${ }^{1}$, Nathalia V. Batista ${ }^{1}$, Kuan Chung Wang ${ }^{1}$, Angela C. Zhou ${ }^{1}$ and Tania H. Watts ${ }^{1}$
}

T-cell responses in the lung are critical for protection against respiratory pathogens. TNFR superfamily members play important roles in providing survival signals to $T$ cells during respiratory infections. However, whether these signals take place mainly during priming in the secondary lymphoid organs and/or in the peripheral tissues remains unknown. Here we show that under conditions of competition, GITR provides a T-cell intrinsic advantage to both CD4 and CD8 effector T cells in the lung tissue, as well as for the formation of CD4 and CD8 tissue-resident memory T cells during respiratory influenza infection in mice. In contrast, under noncompetitive conditions, GITR has a preferential effect on CD8 over CD4 T cells. The nucleoprotein-specific CD8 T-cell response partially compensated for GITR deficiency by expansion of higher affinity T cells; whereas, the polymerase-specific response was less flexible and more GITR dependent. Following influenza infection, GITR is expressed on lung T cells and GITRL is preferentially expressed on lung monocyte-derived inflammatory antigen presenting cells. Accordingly, we show that GITR $+/+T$ cells in the lung parenchyma express more phosphorylated-ribosomal protein S6 than their GITR-/- counterparts. Thus, GITR signaling within the lung tissue critically regulates effector and tissue-resident memory T-cell accumulation.

Mucosal Immunology (2019) 12:363-377; https://doi.org/10.1038/s41385-018-0105-5

\section{INTRODUCTION}

Influenza remains an important human pathogen. CD4 and CD8 T cells play crucial roles in control of influenza virus. ${ }^{1,2}$ However, their activation must be tightly regulated in order to clear the infection, avoid pathology, and at the same time allow the appropriate formation of memory $\mathrm{T}$ cells. ${ }^{3,4}$ The initial activation of $\mathrm{T}$ cells requires an antigen bound to MHC (signal 1), a costimulatory signal through CD28 (signal 2$)^{5}$ as well as cytokines (signal 3). ${ }^{6}$ Although these signals are important in initiating T-cell responses, additional post-priming signals from tumor necrosis factor receptor (TNFR) superfamily members play critical roles in controlling the duration of the T-cell responses, ${ }^{7-12}$ referred to as signal 4 . $^{7}$

Glucocorticoid-induced TNFR-related protein (GITR, TNFRSF18, or CD357), a costimulatory member of the TNFR superfamily plays an important role in the control of viral infections, including influenza virus and LCMV Clone $13^{7,13-15}$ GITR is constitutively expressed at low levels on resting $T$ cells and at high levels on $\mathrm{CD}^{+} \mathrm{CD}^{+} 5^{+}$regulatory $\mathrm{T}$ cells (Treg), ${ }^{16,17}$ and is rapidly upregulated on CD4 and CD8 effector T cells upon activation. ${ }^{16,17}$ GITR is also detected on other immune cell types such as B cells, NK cells, and macrophages. ${ }^{18}$ GITR ligand (GITRL) is mainly expressed on antigen presenting cells (APCs) and endothelial cells, ${ }^{19}$ with monocyte-derived inflammatory APCs showing the highest level of expression during chronic LCMV infection.?

The role of GITR during viral infection appears context dependent. In the chronic LCMV clone 13 infection model, GITR is mainly required on CD4 $T$ cells to provide a post-priming checkpoint for CD4 accumulation and shows only indirect effects on CD8 T cells. ${ }^{7,14}$ In contrast, in a T-cell adoptive transfer model of influenza infection, GITR was shown to mainly affect CD8 T-cell responses, with critical effects on viral control. ${ }^{13}$ However, the role of GITR in the endogenous T cell responses to influenza virus, and when and where these signals take place are incompletely defined. Here, we provide evidence that GITRL on monocytederived inflammatory APCs provides critical signals through GITR on T cells in the mediastinal lymph node $(\mathrm{mLN})$ and then again in the lung tissue to allow effector T-cell accumulation during respiratory influenza infection. This signal is crucial for rescuing low-affinity $\mathrm{D}^{\mathrm{b}} / \mathrm{NP}_{366-374^{-} \text {-specific CD8 T cells as well as for optimal }}$ formation of tissue-resident memory $T$ cells (Trm) in the lung.

\section{RESULTS}

GITR is intrinsically required for effector CD4 and CD8 T-cell accumulation during influenza infection

To determine the intrinsic role of GITR on endogenous CD4 and CD8 T-cell responses during influenza infection, we generated mixed bone marrow chimeras in which Thy 1.1 CD45.2 hosts were lethally irradiated and reconstituted with bone marrow from Thy1.2 CD45.1 GITR ${ }^{+/+}$and Thy1.2 CD45.2 $\mathrm{GITR}^{-/-}$mice mixed in a 1:1 ratio. This model allows us to assess the competition between $\mathrm{GITR}^{+/+}$and $\mathrm{GITR}^{-/-}$cells within the same mouse (Fig. 1a, b). Mice were allowed to reconstitute for 90 days and then pre-infection ratios of donor CD45.1 and CD45.2 were determined in the blood. In another set of bone marrow chimeras, we

Department of Immunology, University of Toronto, Toronto, ON, Canada

Correspondence: Tania H. Watts (tania.watts@utoronto.ca)

Received: 19 March 2018 Revised: 2 October 2018 Accepted: 27 October 2018

Published online: 28 November 2018 
a

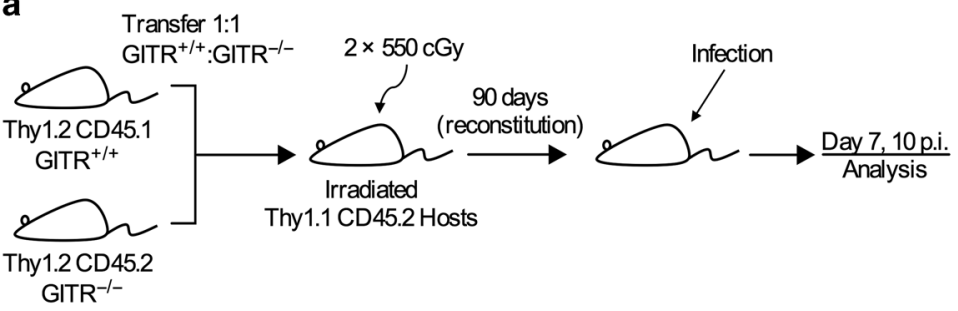

C

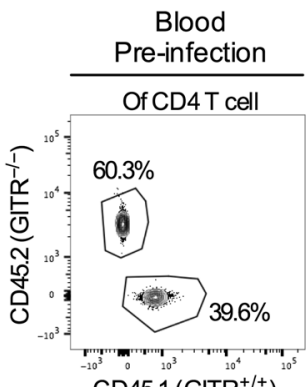

$\operatorname{CD} 45.1\left(\operatorname{GITR}^{+/+}\right)$

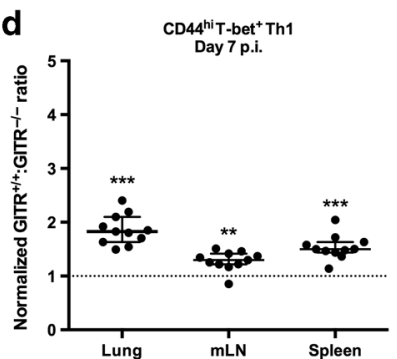

$\mathbf{f}$
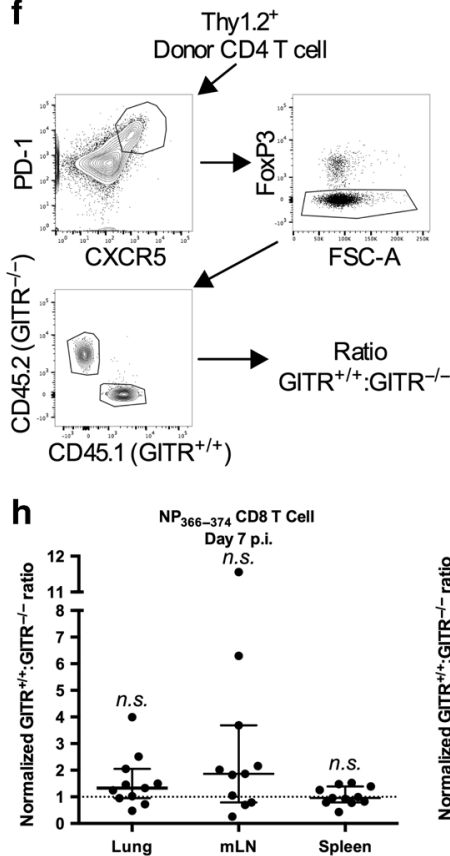

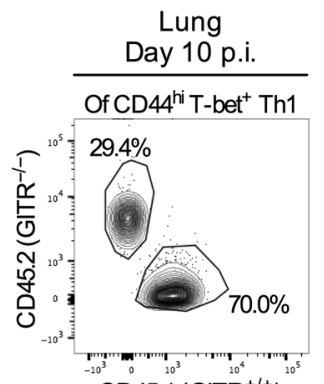

$\operatorname{CD} 45.1\left(\mathrm{GITR}^{+/+}\right)$
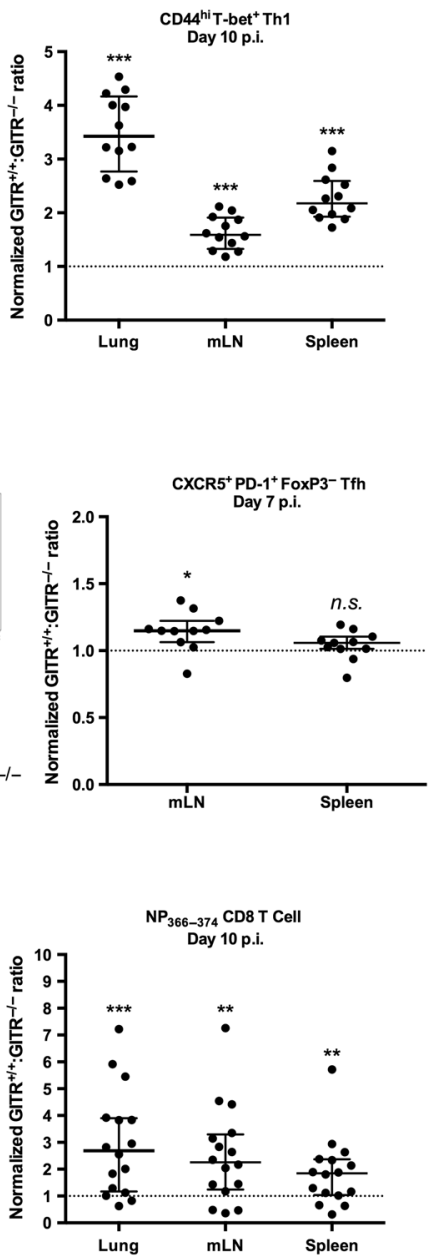

b

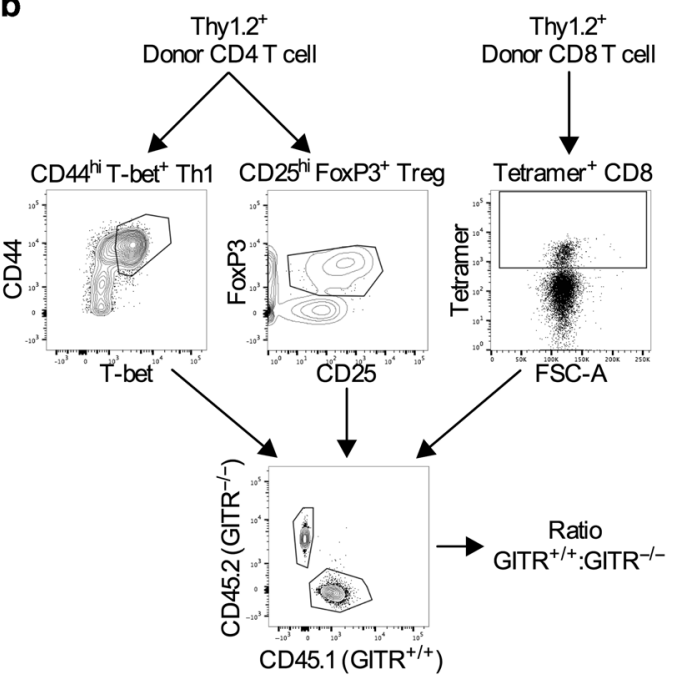

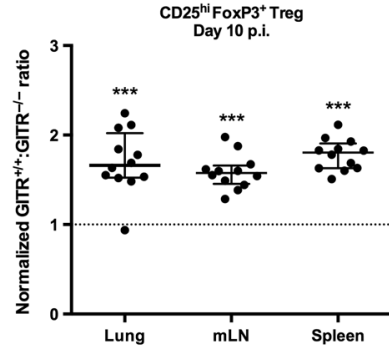

g

Blood

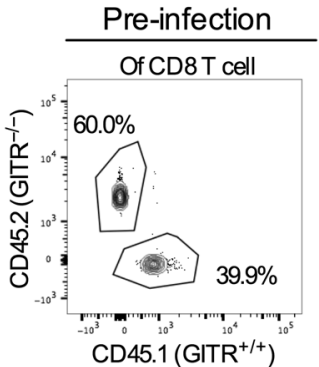

$\mathrm{PA}_{224-233} \mathrm{CDB}$ T Cell

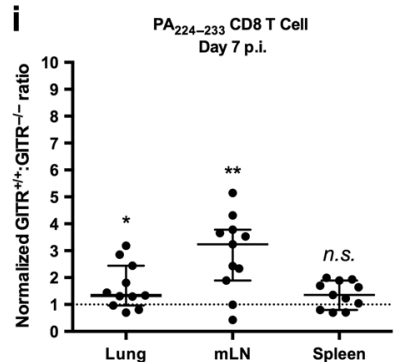

Lung

Day 10 p.i.
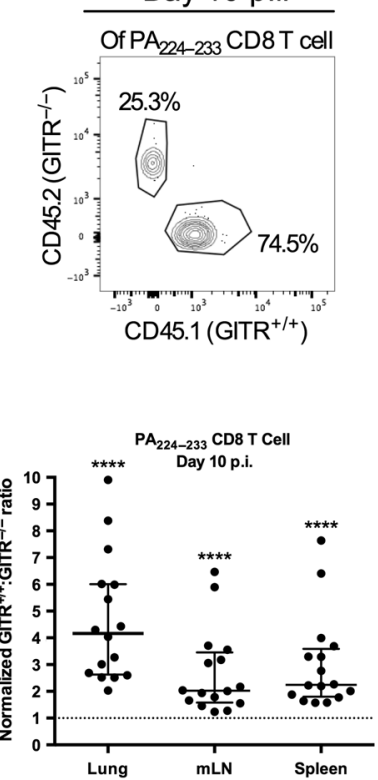

analyzed the reconstitution ratio of $\mathrm{GITR}^{+/+}$to $\mathrm{GITR}^{-/-} \mathrm{CD} 4$ or CD8 $\mathrm{T}$ cells in the spleen and $\mathrm{mLN}$ and found that these ratios were indistinguishable from the ratio measured in the blood (data not shown). Therefore, for each mouse, normalization was done using the pre-infection ratio of $\mathrm{GITR}^{+/+}$to $\mathrm{GITR}^{-/-} \mathrm{T}$ cells measured in the blood. Following infection with influenza A/HK-
X31, GITR ${ }^{+/+}$Th1 cells in the reconstituted mice showed a competitive advantage over $\mathrm{GITR}^{-/-}$Th1 cells in the lung, $\mathrm{mLN}$ and spleen, with a more dramatic effect in the lung (Fig. 1c, d). These effects were greater at the peak of the response, day 10 post infection (p.i.), compared to day 7 p.i. $\mathrm{GITR}^{+/+}$Tregs had a 1.5- to 2-fold advantage over $\mathrm{GITR}^{-1-}$ Tregs in the three organs 
Fig. 1 Under conditions of competition, GITR is intrinsically required for the accumulation of effector CD4 and CD8 T cells during influenza

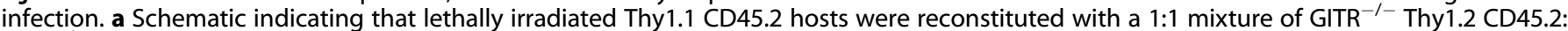
$\mathrm{GITR}^{+/+}$Thy1.2 CD45.1 bone marrow cells. Chimeric mice were rested for 90 days and chimerism checked in the blood of each mouse, before intranasal (i.n.) infection with influenza A/HK-X31 followed by analysis at day 7 or day 10 post infection (p.i.). b Representative gating strategy for donor CD44 ${ }^{\text {hi }}$ T-bet $^{+}$Th1, CD25 $5^{\text {hi }}$ FoxP3 $^{+}$Treg, and tetramer ${ }^{+}$CD8 T cells. c Representative flow cytometry plots showing proportions of GITR $^{+/+}$: GITR ${ }^{-/-}$of blood CD4 T cells before infection and of lung Th1 day 10 p.i. The normalized GITR ${ }^{+/+}:$GITR $^{-/-}$ratio in Th1 d and Treg e compartments was evaluated in the lung, mediastinal lymph node $(\mathrm{mLN})$, and spleen day 7 or day 10 p.i. Normalization was done by dividing the post-infection ratio in each mouse in each tissue by the pre-infection ratio of CD4 T cells in blood from the same mouse. $f$ The normalized $\mathrm{GITR}^{+/+}$: GITR $^{-1-}$ ratio in CXCR5 ${ }^{+}$PD- ${ }^{+}$FoxP3 ${ }^{-}$Tfh compartment was evaluated in the $\mathrm{mLN}$ and spleen day 7 p.i. and normalization was done as described above. Representative gating for donor Tfh is shown on the left. $g$ Representative flow cytometry plots showing proportions of $\mathrm{GITR}^{+/+}:$GITR $^{-/-}$of blood CD8 T cells before infection and of lung $\mathrm{D}^{\mathrm{b}} / \mathrm{PA}_{224-233^{-}}$specific CD8 T cells day 10 p.i. The normalized GITR ${ }^{+/+}: \mathrm{GITR}^{-/-}$

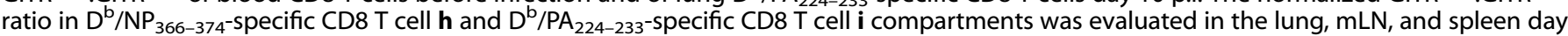
7 or day 10 p.i. Normalization was done using pre-infection ratio of blood CD8 T cells as described for CD4 T cells above. Each symbol represents an individual mouse, with bars indicating median with interquartile range (IQR). Statistical analyses performed using Wilcoxon test comparing pre- and post-infection ratios. Data are pooled from 11 to 16 individual chimeric mice from at least two independent experiments

a

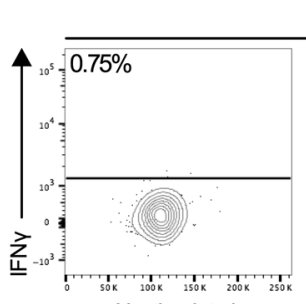

Unstimulated

C

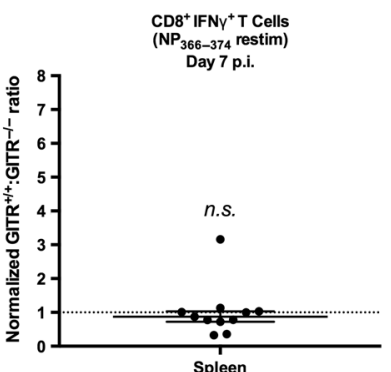

Lung, Day 7 p.i.

Of CD4T cell

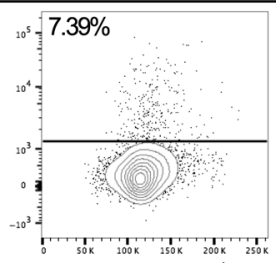

CD45.1 $\mathrm{GTR}^{+/+}$

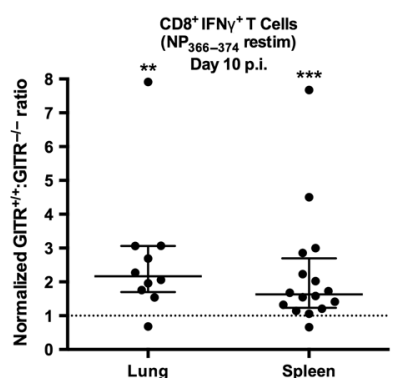

b

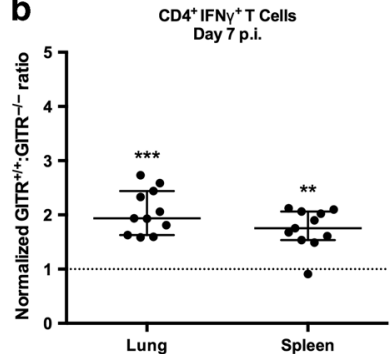

d

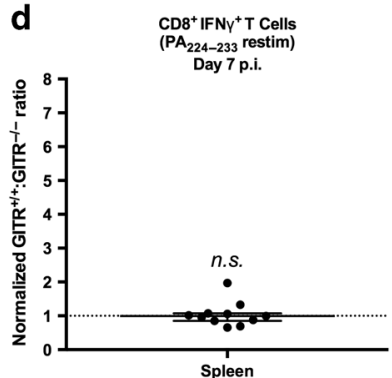

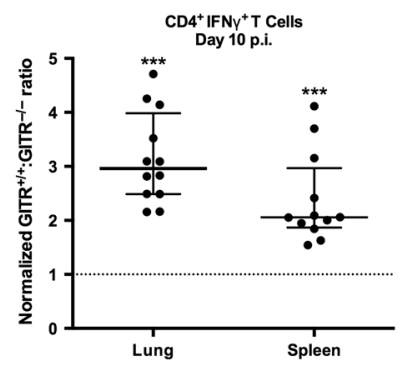

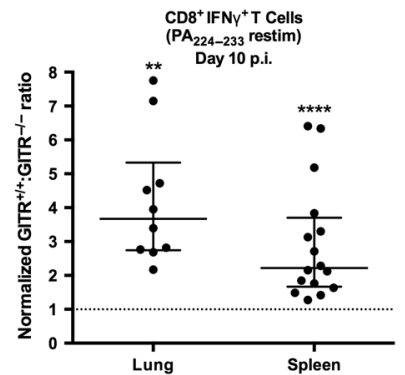

Fig. 2 GITR is intrinsically required for the accumulation of IFN $\gamma$-producing CD4 and CD8 T cells during influenza infection. Mixed bone marrow chimeras were generated and infected as described in Fig. 1. Freshly isolated cells from the lung and splenocytes from chimeric mice were restimulated with live influenza virus for $18 \mathrm{~h}$ a, b or with $D^{5}$-restricted influenza peptides: $\mathrm{NP}_{366-374}$ or $\mathrm{PA}_{224-233}$ for $6 \mathrm{~h} \mathbf{c}$, $\mathbf{d}$, after which intracellular cytokines were measured by flow cytometry. a Representative flow cytometry plots depicting CD4 ${ }^{+} \mathrm{IFN} \gamma^{+} \mathrm{T}_{\text {cells }}$ in the $\mathrm{GITR}^{+/+}$ and GITR ${ }^{-1-}$ compartments day 7 p.i. in the lung. The normalized $\mathrm{GITR}^{+/+}: \mathrm{GITR}^{-/-}$ratio in $\mathrm{CD}^{+}{ }^{+} \mathrm{IFN} \gamma^{+} \mathrm{T}$ cell b, CD8 ${ }^{+}$IFN $\gamma^{+} \mathrm{T}$ cell $(\mathrm{NP}$ restim) $\mathbf{c}$, and $\mathrm{CD}^{+} \mathrm{IFN} \gamma^{+}$T cell (PA restim) $\mathbf{d}$ compartments was evaluated at day 7 or day 10 p.i. in the lung and spleen. Normalization was done as described in Fig. 1. Each symbol represents an individual mouse, with bars indicating median with IQR. Statistical analyses performed using Wilcoxon test comparing pre- and post-infection ratios. Data were pooled from 10 to 16 individual chimeric mice from at least two independent experiments

analyzed at day 7 p.i. with a similar result at day 10 p.i. (Fig. 1e). In contrast, there was little or no effect of GITR on CXCR5 ${ }^{+}$PD-1 $1^{+}$ FoxP3 ${ }^{-}$T follicular helper (Tfh) cells (Fig. 1f).

The effects of GITR on expansion of T-cells specific for the two immunodominant CD8 epitopes of influenza virus in $B 6$ mice, $D^{\mathrm{b}}$ /

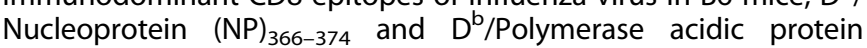
(PA) 224-233, were also more apparent at day 10 than at day 7 with the PA epitope showing more sensitivity to GITR than the NP

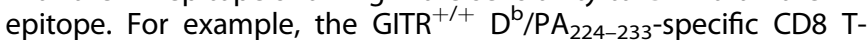
cells exhibited a 4.2-fold advantage over their $\mathrm{GITR}^{-1-}$ counterparts, compared to a 2.7 -fold for $\mathrm{GITR}^{+/+} \mathrm{D}^{\mathrm{b}} / \mathrm{NP}_{366-374}$-specific CD8 T cells at day 10 p.i. in the lung (Fig. $1 \mathrm{~g}-\mathrm{i}, p=0.021$, Wilcoxon Test).

$\mathrm{GITR}^{+/+}$IFNy-producing CD4 T-cells detected after whole virus restimulation ex vivo also showed increased proportions compared to their GITR ${ }^{-/-}$counterparts (Fig. 2a, b) and similar results were obtained with IFNY-producing CD8 $\mathrm{T}$ cells after restimulation with $\mathrm{NP}_{366-374}$ or $\mathrm{PA}_{224-233}$ peptides (Fig. 2c, d). GITR had a significantly greater effect on accumulation of PA- as compared to
NP-specific IFN- $\gamma$-producing cells (comparison of fold effect of GITR on NP vs PA Lung day 10 p.i., $p=0.029$ Mann-Whitney Test).

Thus, in a competitive setting, GITR is intrinsically required for the accumulation of Th1 cells and antigen-specific CD8 T cells, but minimally affects Th cells during influenza infection and $D^{\mathrm{b}}$ / $\mathrm{PA}_{224-233}$-specific CD8 T cells were more dependent on GITR than $\mathrm{D}^{\mathrm{b}} / \mathrm{NP}_{366-374}$-specific CD8 T cells.

GITR signaling rescues low-affinity $\mathrm{D}^{\mathrm{b}} / \mathrm{NP}_{366-374^{-}}$specific CD8 T cells

During an immune response, multiple factors determine which $\mathrm{T}$ cells among a polyclonal repertoire expand and dominate the effector response. One such factor is access to costimulatory signals. ${ }^{20,21}$ For example, the TNFR family member CD27 plays a key role in rescuing low-affinity CD8 T cells during influenza infection. ${ }^{21}$ Previous studies have established that $\mathrm{T}$ cell receptor (TCR) affinity strongly correlates with the amount of tetramer binding. ${ }^{21-23} \mathrm{~A}$ higher affinity $\mathrm{T}$ cell will bind more tetramer than a low-affinity $T$ cell, due to the lower valency requirement for the 

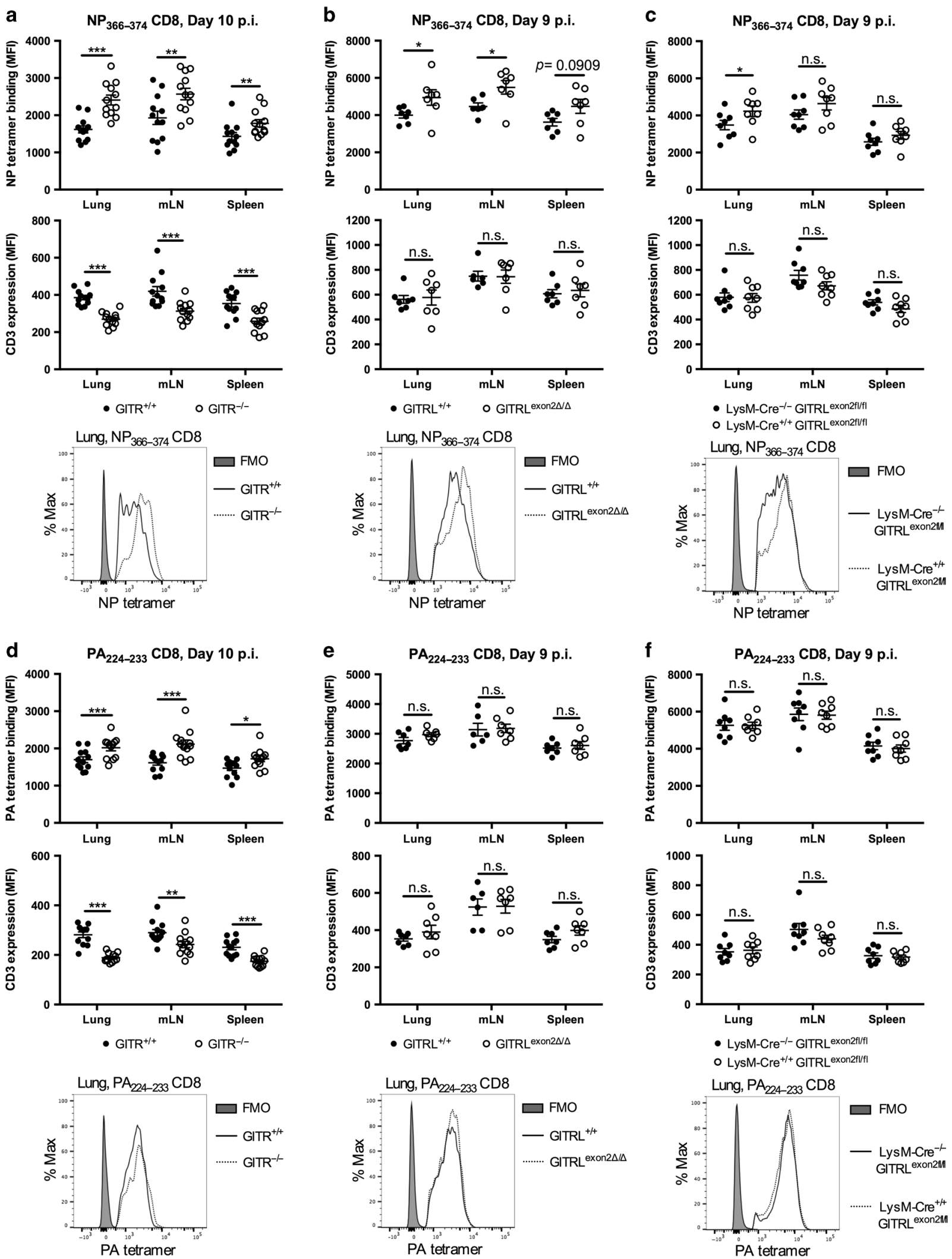

tetramers to bind to the TCR. Accordingly, we analyzed the level of tetramer binding per cell to ask whether GITR affects the affinity of

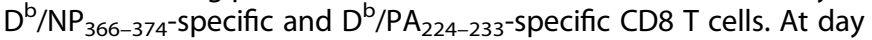
10 p.i. of the mixed bone marrow chimeras, we observed a significant increase in the median fluorescence intensity (MFI) of
NP tetramer bound to $\mathrm{GITR}^{-/-} \mathrm{D}^{\mathrm{b}} / \mathrm{NP}_{366-374}$-specific CD8 T cells in the lung, spleen and $\mathrm{mLN}$, when compared to their $\mathrm{GITR}^{+/+}$ counterparts (Fig. 3a). This was not due to a higher level of T-cell receptor per cell, as we observed even lower CD3 levels on $\mathrm{GITR}^{-1-} \mathrm{D}^{\mathrm{b}} / \mathrm{NP}_{366-374}$-specific CD8 $\mathrm{T}$ cells compared to their 
Fig. 3 GITR costimulation rescues low-affinity $\mathrm{D}^{\mathrm{b}} / \mathrm{NP}_{366-374}$-specific CD8 T cells. a, $\mathbf{d} \mathrm{GITR}^{+/+}$:GITR ${ }^{-/-}$mixed bone marrow chimeras were generated and infected as described in Fig. 1. b, e Wild-type C57BL/6 (GITRL ${ }^{+1+}$ ) mice were co-housed with GITRL exon2 $2 / \Delta$ mice from weaning and infected i.n. with influenza A/HK-X31 and analyses were done at day 9 p.i. c, $\mathbf{f}$ LysM-Cre ${ }^{+/+}$and LysM-Cre $^{-/-}$GITRL $^{\text {exon2fl/fl }}$ littermates were

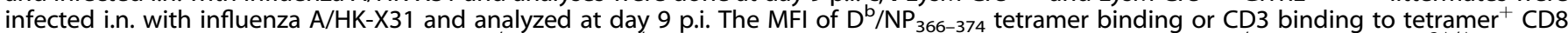
T cells in the lung, $\mathrm{mLN}$, and spleen of $\mathrm{GITR}^{+/+}$and $\mathrm{GITR}^{-/-}$donor origin was determined on day 10 a, of GITRL ${ }^{+/+}$and GITRL ${ }^{\text {exon } 2 \Delta / \Delta}$ mice on day 9 b, and of LysM-Cre ${ }^{+/+}$and LysM-Cre ${ }^{-/-}$GITRL ${ }^{\text {exon2fl/fl }}$ mice on day 9 c. MFI of $D^{\mathrm{b}} / \mathrm{PA}_{224-233}$ tetramer binding or CD3 binding of tetramer ${ }^{+}$ CD8 T cells in the lung, $\mathrm{mLN}$, and spleen of $\mathrm{GITR}^{+/+}$and GITR ${ }^{-/-}$donor cells was determined on day $10 \mathrm{~d}, \mathrm{of} \mathrm{GITRL}^{+/+}$and GITRL exon2 $2 / \Delta$ mice on day $9 \mathbf{e}$, and of LysM-Cre ${ }^{+/+}$and LysM-Cre ${ }^{-/-}$GITRL $^{\text {exonzfl/fl }}$ mice on day 9 f. Representative histogram plots are shown below the summary plots for each set of data. Each symbol represents an individual mouse, with bars indicating mean \pm SEM. Statistical analyses performed using Wilcoxon test $\mathbf{a}$ and $\mathbf{d}$ or Mann-Whitney test $\mathbf{b}, \mathbf{c}, \mathbf{e}$, and $\mathbf{f}$. Data from $\mathbf{a}$ and $\mathbf{d}$ pooled from 12 individual chimeric mice with two independent experiments. Data from $\mathbf{b}$ and $\mathbf{e}$ were pooled from three independent experiments with a total of 6-7 mice per group. Data from $\mathbf{c}$ and $\mathbf{f}$ were pooled from two independent experiments with a total of eight mice per group

$\mathrm{GITR}^{+/+}$counterparts in the same mouse (Fig. 3a). Similarly, D

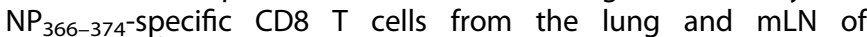
GITRL ${ }^{\text {exon } 2 \Delta / \Delta}$ mice bound more tetramer than $T$ cells from $\mathrm{GITRL}^{+/+}$mice, whereas there was no change in the CD3 level (Fig. 3b). A similar effect was seen in the lung with mice lacking exon 2 of GITRL only in myeloid cells (LysM+ cells) compared to their Cre-negative littermates (Fig. 3c). GITRL ${ }^{\text {exon2 } 2 / \Delta}$ mice lack an 11 amino acid sequence encoded by exon 2 , resulting in a thermally unstable protein and a partial defect in activation of GITR signaling.

In contrast to the results with the NP epitope-specific T cells, there was only a minimal, albeit significant, increase in the amount of PA tetramer bound to $\mathrm{GITR}^{-1-} \mathrm{D}^{\mathrm{b}} / \mathrm{PA}_{224-233^{-} \text {specific CD8 T cells }}$ when compared to their wild-type counterparts (Fig. 3d). Moreover, there was no difference in the amount of PA tetramer bound to $D^{\mathrm{b}} / \mathrm{PA}_{224-233^{-}}$specific CD8 $\mathrm{T}$ cells between GITRL ${ }^{\text {exon } 2 \Delta / \Delta}$ and $\mathrm{GITRL}^{+/+}$mice (Fig. 3e) or LysM-Cre ${ }^{+/+}$and $\mathrm{LysM}^{-C r e}{ }^{-/-}$ GITRL $^{\text {exon2fl/fl }}$ littermate mice (Fig. 3f).

Together, these results suggest that GITR allows the accumulation of low-affinity $D^{\mathrm{b}} / \mathrm{NP}_{366-374}$-specific effector CD8 T cells during influenza infection and this is largely due to GITRL on $\mathrm{LysM}^{+}$myeloid cells. In contrast, the PA-epitope-specific $\mathrm{T}$ cells, despite more costimulation-dependence, show only a minimal difference in tetramer binding on $\mathrm{GITR}^{+/+}$and $\mathrm{GITR}^{-/-}$ $T$ cells, suggesting similar affinity. This difference between the NP and PA epitope-specific T cells was most evident in the lung (Fig. 3a, d).

GITR is required for the optimal formation of lung CD4 and CD8 Trm following influenza infection

CD4 and CD8 Trm, subsets of highly protective non-circulating memory $T$ cells that reside in the peripheral tissue, are critical for protective immunity to influenza infection. ${ }^{24-27} \mathrm{~A}$ recent study showed that the TNFR family member 4-1BB critically contributes to lung Trm formation. ${ }^{28}$ To investigate, whether GITR is also required for the optimal establishment of lung CD4 and CD8 Trm following influenza infection, we again used competitive mixed bone marrow chimeras where GITR ${ }^{+/+}$and GITR ${ }^{-1-}$ cells compete within the same mouse (Fig. 4a). Prior to harvest, we used intravascular infusion of fluorescently labeled anti-Thy 1.2 to distinguish lung vascular (LV) exposed cells from cells located within the lung tissue (LT). ${ }^{29,30}$ CD4 Trm were defined based on CD69 and CD11a co-expression and CD8 Trm based on CD69 and CD103 co-expression (Fig. 4b). At day 30 p.i., GITR ${ }^{+/+}$LT CD4 Trm had a fivefold advantage over their GITR $^{-1-}$ counterparts, whereas $\mathrm{GITR}^{+/+}$and $\mathrm{GITR}^{-/-}$LV CD4 T cells competed equally well (Fig. 4c, d). Similarly, in the tissue, the total GITR ${ }^{+/+}$CD8 Trm, as well as the tetramer positive subpopulations had a 3- to 3.5-fold advantage over their $\mathrm{GITR}^{-1-}$ counterparts, whereas the CD8 $T$ cells in the vasculature showed no such difference (Fig. 4c, e). GITR did not influence the number of CD4 or CD8 Trm in the spleen (data not shown). In fact, there were very few NP-specific CD8 Trm detectable in the spleen. Moreover, GITR had a less dramatic effect on splenic CD4 and CD8 central memory T cells (Tcm) and effector memory $T$ cells (Tem) than on the lung Trm (Fig. 4f, g). In sum, GITR is required for the optimal formation of lung CD4 and CD8 Trm.

Under non-competitive conditions, GITR has a preferential role on CD8 over CD4 T-cell responses to influenza virus

To determine whether GITR was also required for T-cell responses in a non-competitive setting, we infected $\mathrm{GITR}^{+/+}$and $\mathrm{GITR}^{-/-}$ littermate mice with influenza A/HK-X31 and assessed CD4 and CD8 T-cell responses. At day 9 p.i., there was a significant reduction in the total number of CD8 T cells in the lung, as well as $\mathrm{D}^{\mathrm{b}} / \mathrm{NP}_{366-374^{-}}$-specific and $\mathrm{D}^{\mathrm{b}} / \mathrm{PA}_{224-233^{-}}$-specific $\mathrm{CD} 8 \mathrm{~T}$ cells in $\mathrm{GITR}^{-1-}$ mice when compared to their GITR ${ }^{+/+}$littermates (Fig. 5a), whereas there was no deficit in $\mathrm{GITR}^{-1-}$ T-cell responses in the $\mathrm{mLN}$ and spleen (Supplementary Fig. S1a). At day 30 p.i., there was a significant reduction in the total number of polyclonal $\mathrm{CD}^{+} 9^{+}$ $\mathrm{CD}_{103}{ }^{+} \mathrm{CD} 8 \mathrm{Trm}$ in the lung parenchyma, as well as $\mathrm{D}^{\mathrm{b}} / \mathrm{PA}_{224-233^{-}}$ specific CD8 Trm in GITR ${ }^{-1-}$ mice when compared to $\mathrm{GITR}^{+/+}$ littermates (Fig. 5b). GITR ${ }^{-1-}$ mice exhibited a trend towards fewer

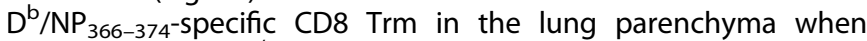
compared to GITR $^{+/+}$littermates (Fig. 5 b). In contrast to the results from the mixed bone marrow chimeras, there was no difference in the total number of CD4 T cells, Th1, and Treg in the lung, $\mathrm{mLN}$, and spleen between GITR $^{+/+}$and GITR $^{-1-}$ mice (Fig. 5c and Supplementary Fig. S1b). Similarly, at day 30 p.i., there was no significant difference in the number of lung parenchymal $\mathrm{CD}_{69}{ }^{+}$ CD11a ${ }^{\text {hi }}$ CD4 Trm between GITR ${ }^{+/+}$and GITR ${ }^{-/-}$mice (Fig. $5 \mathrm{~d}$ ). Again, the Tfh response in the $\mathrm{mLN}$ and spleen of $\mathrm{GITR}^{-1-}$ mice showed no detectable differences when compared to $\mathrm{GITR}^{+/+}$ mice (Fig. 5e). These data show that while GITR is required for optimal lung CD8 effector and resident memory $\mathrm{T}$-cell responses against influenza under both competitive and non-competitive conditions, the effects of GITR on CD4 T-cell responses are evident only under conditions of competition.

GITR is transiently upregulated on Th1, Treg, and influenza-specific CD8 $T$ cells in the lung and $\mathrm{mLN}$

To gain insight into where GITR costimulation takes place, we examined the expression of GITR on T cells isolated from the $\mathrm{mLN}$ and the lung during influenza infection. At day 3 p.i. in the $m L N$, activated effector CD4 T cells (CD44 ${ }^{\text {hi }} \mathrm{CD} 9^{+}$FoxP3 $^{-}$) and CD8 $\mathrm{T}$ cells $\left(\mathrm{CD} 44^{\mathrm{hi}} \mathrm{CD69}^{+}\right.$) upregulated GITR relative to naive controls (Fig. 6a, b). Consistent with previously published results, ${ }^{16,17}$ Treg had the highest GITR expression in the $\mathrm{mLN}$ and the lung (Fig. 6a, C). We observed GITR expression on the total lung CD4 T-cell population as well as the Th1 population at day 5 p.i., with peak expression at day 7 p.i., declining again by day 9 p.i. (Fig. $6 \mathrm{c}$ ). $\mathrm{D}^{\mathrm{b}}$ /

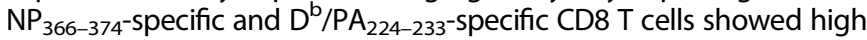
GITR expression at day 5 p.i. in the lung, declining by day 7, and returning to baseline by day 9 (Fig. 6d). Thus, GITR is transiently upregulated upon T-cell activation and is detected in both the $\mathrm{mLN}$ and the lung, with peak expression at day 5-7 p.i. in the lung. 
a

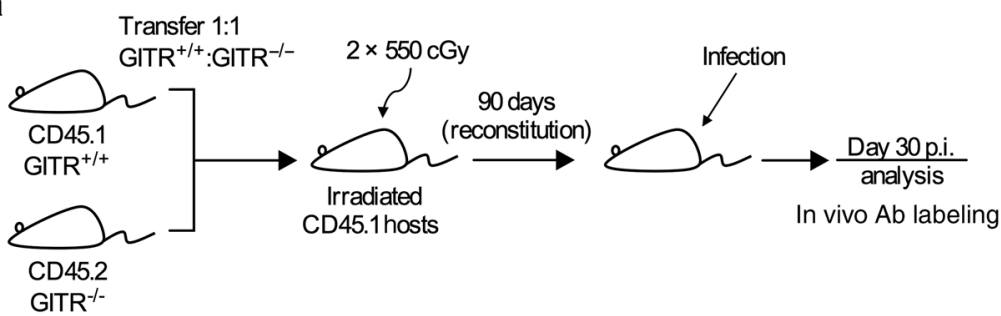

C
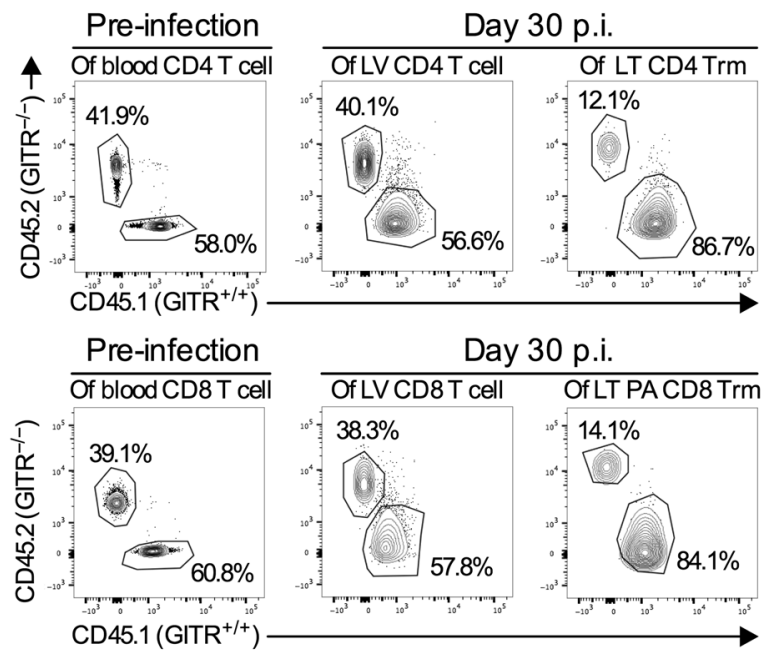

d

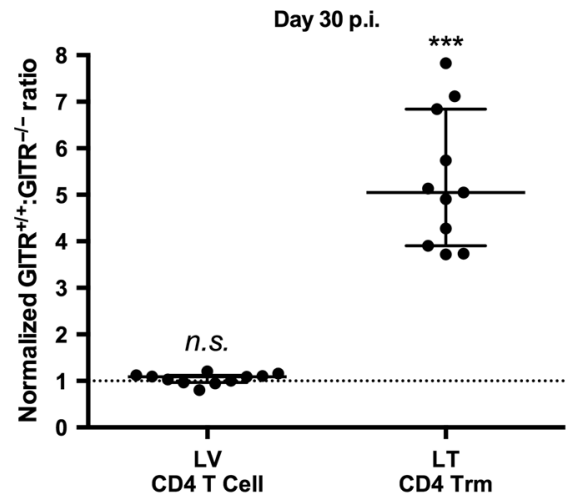

f

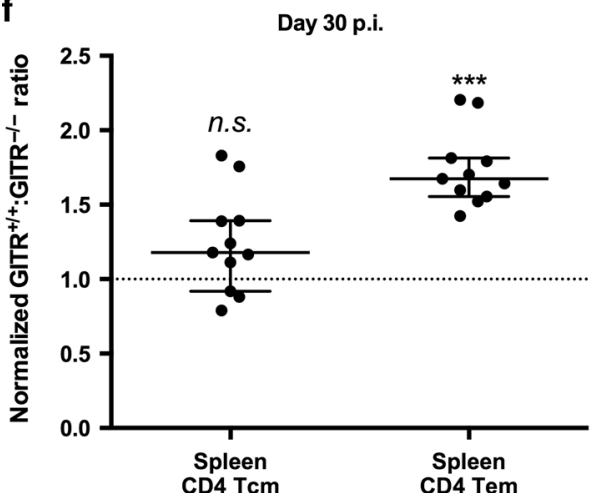

e

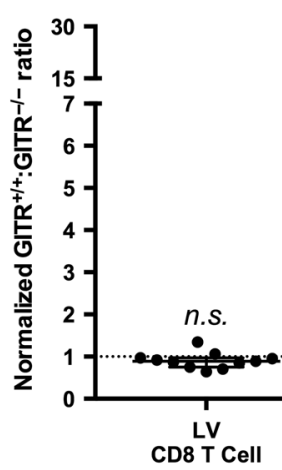

g b Donor CD4/CD8T cell
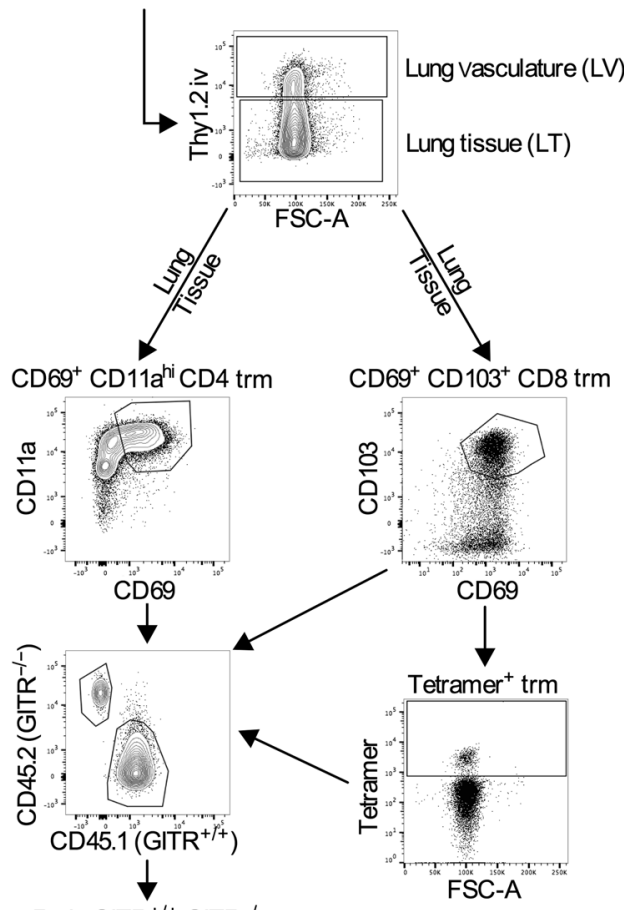

Ratio $\mathrm{GITR}^{+/+}: \mathrm{GITR}^{-/-}$

Day 30 p.i.

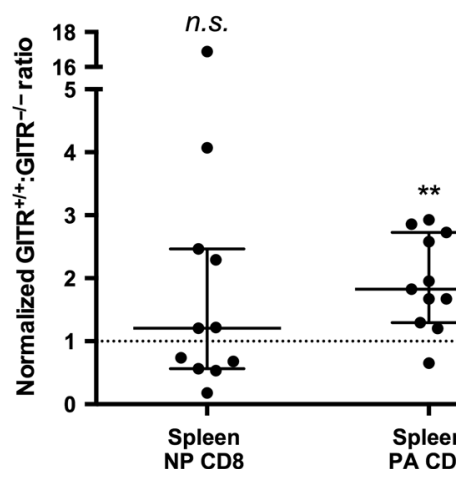

Day 30 p.i.

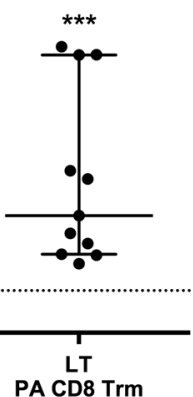

$\bullet$

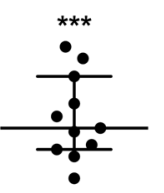

PA CD8 Trm

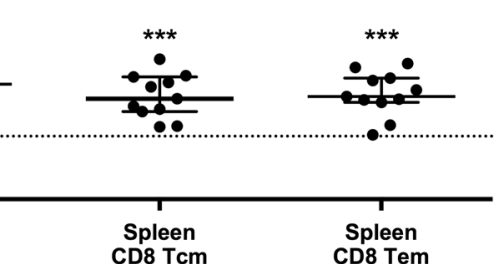

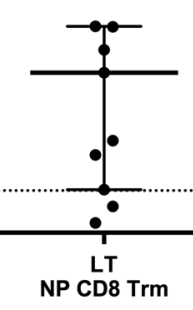

GITRL expression is highest on monocyte-derived inflammatory APC subsets after influenza infection

We next examined the expression of GITRL on immune cell subsets in the lung and $m L N$ from pre-infection (day-1) through the first 8 days of influenza A/HK-X31 infection. A 12-parameter flow cytometry panel was used to delineate different immune cell subsets in the lung and $\mathrm{mLN}$, with emphasis on APC subsets
(Supplementary Fig. S2a, b). Inflammatory DCs (InfDC) and inflammatory macrophages (InfMF) were distinguished from other cell subsets using the high affinity lgE receptor, FceRI (Supplementary Fig. S2a, b). ${ }^{31,32}$ We observed highest GITRL expression on InfDC and InfMF with peak expression day 4 to day 5 p.i. (Fig. 7a). Alveolar macrophages (AlvMF, $\mathrm{CD} 11 \mathrm{c}^{+}$Siglec- $\mathrm{F}^{+}$) also appeared to stain with GITRL. However, examination of the histograms showed 
Fig. 4 GITR is required for the optimal formation of lung CD4 and CD8 Trm after influenza infection. a Schematic indicating that lethally irradiated CD45.1 hosts were reconstituted with a 1:1 mixture of GITR ${ }^{-1-}$ CD45.2: GITR ${ }^{+1+}$ CD45.1 bone marrow cells. Chimeric mice were rested for 90 days and blood chimerism checked before i.n. infection with influenza A/HK-X31 and analyses were done at day 30 p.i. b Representative gating for donor $\mathrm{CD}_{6} 9^{+} \mathrm{CD} 11 \mathrm{a}^{\text {hi }} \mathrm{CD} 4 \mathrm{Trm}$, polyclonal $\mathrm{CD} 69^{+} \mathrm{CD} 103^{+} \mathrm{CD} 8$ Trm, and tetramer ${ }^{+} \mathrm{CD} 8 \mathrm{Trm}$ from the lung parenchyma. c Top: Representative flow cytometry plots showing proportions of GITR ${ }^{+/+}: \mathrm{GITR}^{-/-}$of CD4 T cells in blood before infection, of lung vascular (LV) CD4 T cell at day 30 p.i., and of lung tissue (LT) CD4 Trm at day 30 p.i. Bottom: Representative flow cytometry plots showing

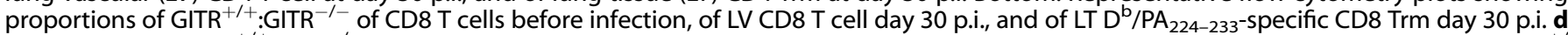
The normalized GITR ${ }^{+/+}: \mathrm{GITR}^{-/-}$ratio in the LV CD4 T cell and LT CD4 Trm compartments shown for individual mice. e The normalized GITR ${ }^{+\prime}$ ${ }^{+}$GITR $^{-1-}$ ratio in the LV CD8 T cell, LT polyclonal CD8 Trm, LT D ${ }^{\mathrm{b}} / \mathrm{NP}_{366-374}$-specific CD8 Trm, and LT D $/$ PA $224-233^{-S p e c i f i c ~ C D 8 ~ T r m ~}$

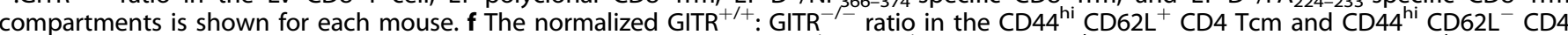

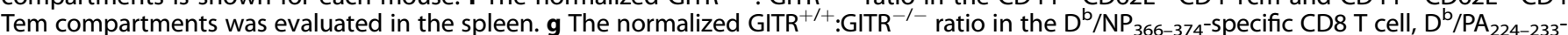
specific CD8 T cell, CD44 ${ }^{\text {hi }} \mathrm{CD}_{2} \mathrm{~L}^{+} \mathrm{CD} 8 \mathrm{Tcm}$ and CD44 ${ }^{\mathrm{hi}} \mathrm{CD} 62 \mathrm{~L}^{-}$CD8 Tem compartments was evaluated in the spleen. Normalization was done as described in Fig. 1. Each symbol represents an individual mouse, with bars indicating median with IQR. Statistical analyses performed using Wilcoxon test comparing pre- and post-infection ratios. Data were pooled from two independent experiments with 11 individual chimeric mice

that the AlvMF have a high-background fluorescence and didn't show a major shift in stain over FMO and the apparent change in MFI is not reflective of significant GITRL expression (Fig. 7a, bottom row). In the $\mathrm{mLN}$, we observed a similar pattern of GITRL expression with InfDC and InfMF exhibiting highest GITRL expression among different immune cell subsets, albeit with slightly earlier kinetics when compared to the lung (Fig. 7b). As InfDC and InfMF had the highest GITRL expression among different APC subsets in the lung, we tracked the number of InfDC and InfMF in the lung over the course of influenza infection. We observed highest numbers of InfDC and InfMF in the lung at day 5 p.i. and the number declined by day 8 p.i. (Fig. 7c). Next, we asked whether a stronger influenza infection model (influenza A/ PR8) would induce higher expression of GITRL. However, we observed a similar pattern of GITRL expression with marginally lower GITRL expression during PR8 infection when compared to X31 infection. Of note, the number of InfDC and InfMF was lower during the more severe PR8 infection, but the inflammatory APC persisted through to day 8 p.i. (Supplementary Fig. S3a-c). B220 PDCA $-1^{+}$plasmacytoid dendritic cells ( $\mathrm{PDC}$ ) were also examined in a separate panel and found to express minimal levels of GITRL in the lung and mLN (Supplementary Fig. S3d and data not shown).

Recent data examining GITRL expression during LCMV clone 13 infection, showed that GITRL is induced on APC by type I interferon (IFN-I). ${ }^{7}$ To determine whether this is also the case in the lung during influenza virus infection, we analyzed InfAPC and classical DCs (CDC) using the markers FceRI, CD64, Ly6C, CD11C, and MHC II (Fig. 8a). Mice were treated with two doses of isotype control or blocking antibodies to the interferon receptor subunit 1 (IFNAR1), administered one day before and on the day of infection, and GITRL expression was assessed in the lung at day 3 p.i. The expression of GITRL during influenza infection was substantially blocked on InfAPC and CDC by anti-IFNAR1 treatment (Fig. 8b). These results show that GITRL expression is highest on InfAPC subsets in both the $\mathrm{mLN}$ and lung during influenza infection, mediated at least in part by IFN-I.

GITR-dependent signals in T cells take place in the $\mathrm{mLN}$ and in the lung tissue

The finding that GITRL was detected early in the $\mathrm{mLN}$ combined with the finding that GITR on T cells and GITRL on lung inflammatory APC are present as the T cells start to accumulate in the lung, suggests that GITR costimulation may first take place in the $\mathrm{mLN}$ and then again in the lung tissue. To more directly test this hypothesis, we analyzed GITR-dependent signaling in T cells from mLN and lung directly ex vivo. Previous studies have shown that GITR signaling in CD4 T cells specifically induces phosphorylated-ribosomal protein S6 (pS6), ${ }^{7,14}$ a downstream target of mTOR that is important in regulation of cell size and glucose homeostasis. ${ }^{33}$ To analyze GITR-dependent induction of
pS6 in the $\mathrm{mLN}$ and lung, we adoptively transferred a 1:1 mixture of $\mathrm{GITR}^{+/+}$and $\mathrm{GITR}^{-1-}$ CD45.1 OT-II cells from littermate mice into wild-type CD45.2 C57BL/6 mice followed by infection with influenza PR8 carrying the OT-II epitope (PR8-OVAll) (Fig. 9a). GITR expression on donor CD45.1 OT-II cells was used to distinguish $\mathrm{GITR}^{+/+}$and GITR ${ }^{-/-} \mathrm{T}$ cells pre- and post-infection (Fig. 9b). By co-transferring $\mathrm{GITR}^{+/+}$and GITR ${ }^{-/-}$OT-II into the same recipient, they are exposed to the same level of the virus and soluble mediators, therefore signals directly downstream of GITR can be determined by comparing GITR ${ }^{+/+}$and $\mathrm{GITR}^{-1-} \mathrm{T}$ cells within each mouse. At day 3 p.i., GITR ${ }^{+/+}$OT-Il cells isolated from the $\mathrm{mLN}$ had a higher level of pS6 than GITR ${ }^{-1-}$ OT-II T cells from the same mice (Fig. 9c). Thus, GITR signaling can occur in the $\mathrm{mLN}$ by day 3 p.i.

We next examined OT-II responses in the lung. At day 5 p.i., there was an equal frequency and total number of $\mathrm{GITR}^{+/+}$and $\mathrm{GITR}^{-/-}$OT-II in the lung (Supplementary Fig. S4a), suggesting that there is no defect in GITR ${ }^{-1-}$ T cells reaching the lung postpriming. However, the GITR ${ }^{+/+}$OT-II cells isolated from the lung had a higher level of pS6 than GITR ${ }^{-1-}$ OT-II T cells from the same mice (Fig. 9d). Similar experiments done with Thy1.2 infusion showed that in the lung parenchyma GITR ${ }^{+/+}$OT-II cells had a higher level of pS6 than GITR ${ }^{-/-}$OT-II cells (data not shown). At day 10 p.i., there was a lower frequency and total number of GITR $^{-/-}$OT-Il cells in the lung, $\mathrm{mLN}$, and spleen than their $\mathrm{GITR}^{+/+}$ counterparts, with a more dramatic result in the lung, similar to what we observed in the bone marrow chimera model (Fig. 9e, f). There was also a lower frequency and total number of IL-2 producing $\mathrm{GITR}^{-1-}$ OT-II in the lung but not in the spleen (Supplementary Fig. S4b-d). At day 10 p.i., there was no difference in the IL-2 production per cell in the lung and there is a marginal but significant decrease in the IL-2 production per cell in GITR ${ }^{-/-}$ OT-II in the spleen (Supplementary Fig. S4e). There was also no difference in the frequency and total number of IFN- $\gamma$ producing OT-II between GITR ${ }^{+/+}$and GITR ${ }^{-1-}$ donor cells (Supplementary Fig. S4b, f). Thus, the decreased pS6 signal in GITR ${ }^{-1-}$ OT-II cells correlated with decreased accumulation of the GITR ${ }^{-1-}$ OT-II cells as well as GITR $^{-1-}$ IL-2 producing OT-II cells compared to their $\mathrm{GITR}^{+/+}$counterparts. These results suggest that GITR costimulation on $T$ cells can occur in the $\mathrm{mLN}$ and then in the lung tissue to promote accumulation of the T cells.

Next, we used a similar approach to assess if GITR costimulation can occur in in CD8 T cells in the lung. We adoptively transferred a 1:1 mixture of $\mathrm{GITR}^{+/+}$and $\mathrm{GITR}^{-/-}$CD45.2 OT-I cells from littermate mice into CD45.1 mice followed by infection with influenza PR8 carrying the OT-I epitope (PR8-OVA) (Fig. 9g). At day 5 p.i., GITR ${ }^{+/+}$OT-I isolated from the lung exhibited a small but significantly higher level of pS6 than GITR ${ }^{-/-}$OT-I T cells from the same mice (Fig. 9h). Thus, GITR signaling on CD8 T cells can also take place in the lung tissue. 
a Lung CD8 T cell, day 9 p.i.
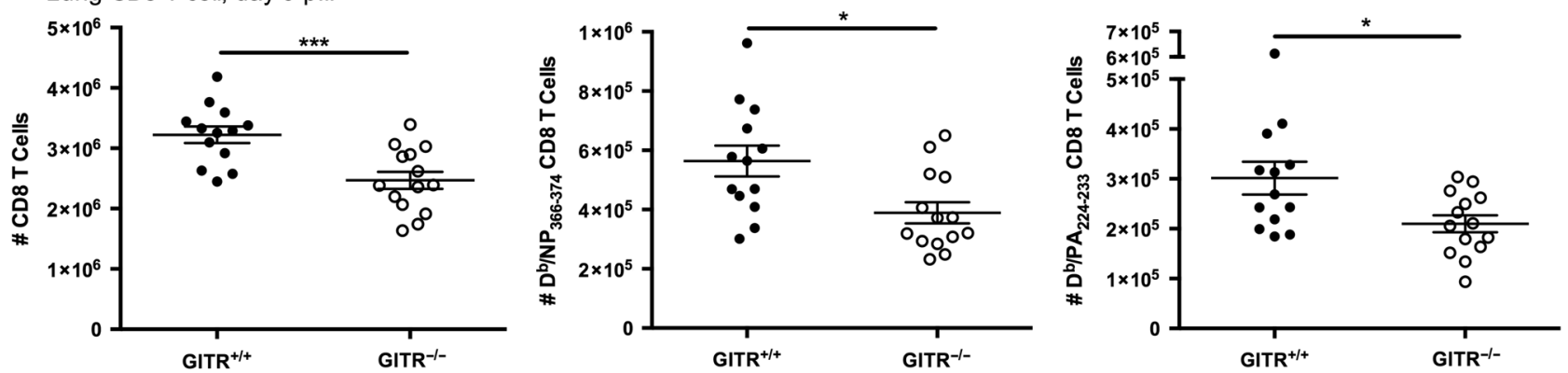

b Lung CD8 Trm, day 30 p.i.
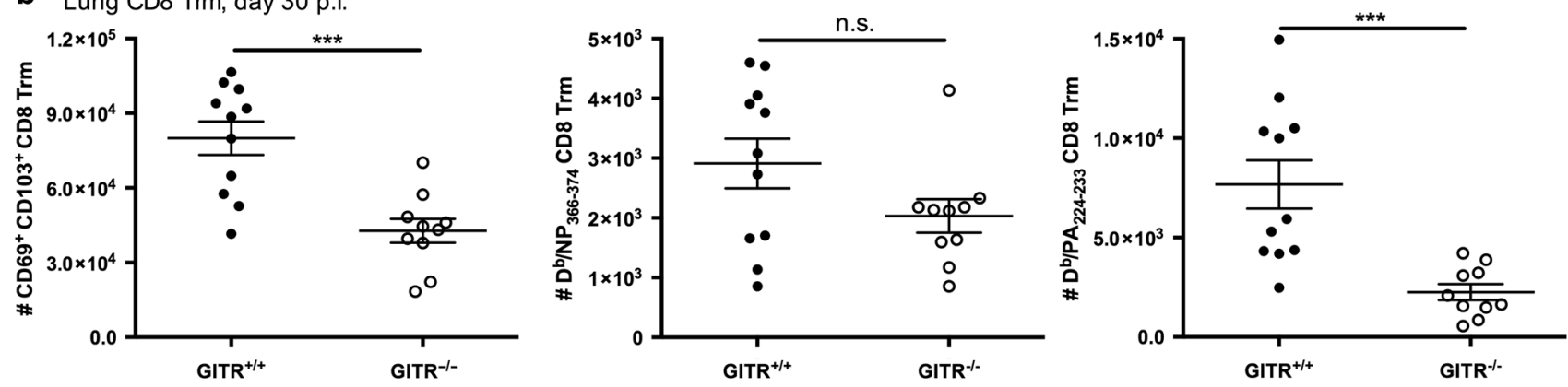

C Lung CD4 T cell, day 9 p.i.
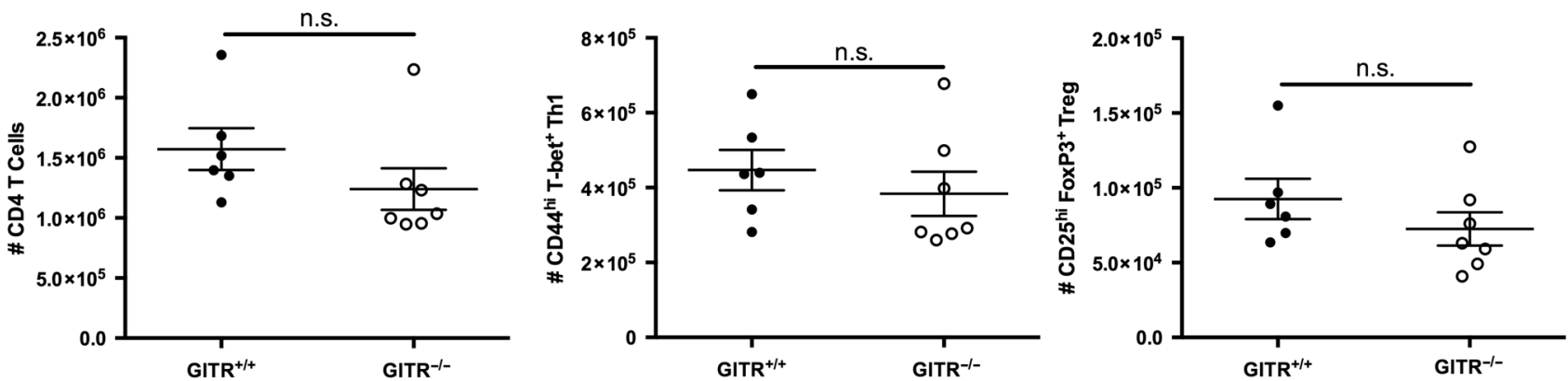

d Lung CD4 Trm, day 30 p.i.

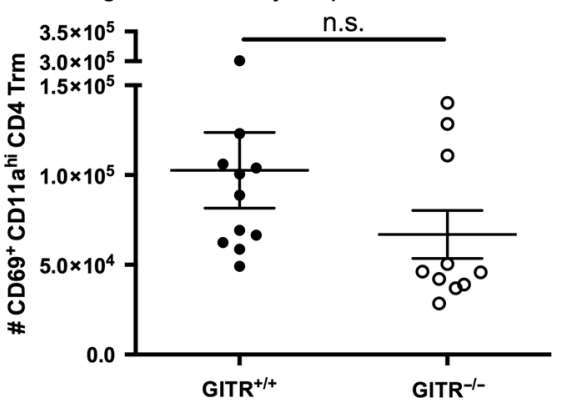

e $\mathrm{mLN}$ Tfh, day 9 p.i.

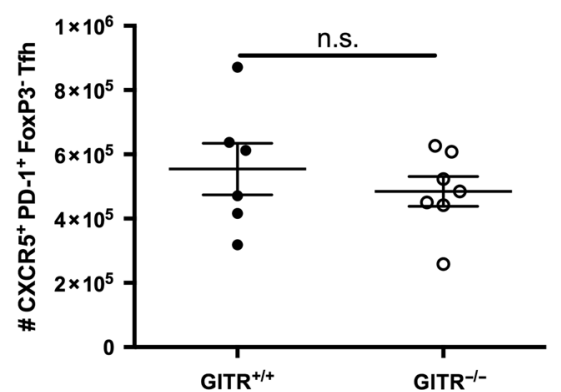

Spleen Tfh, day 9 p.i.

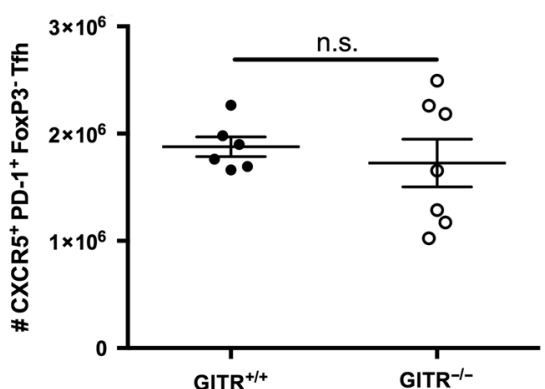

Fig. 5 In the absence of competition, GITR is required for optimal CD8 T-cell response and CD8 Trm formation but dispensable for the CD4 Tcell response and CD4 Trm formation in response to influenza virus. GITR ${ }^{+/+}$and $\mathrm{GITR}^{-/-}$littermates were infected i.n. with influenza A/HK-

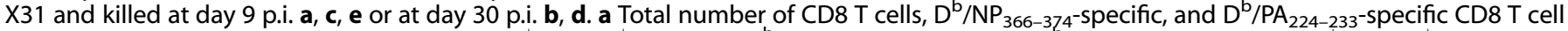

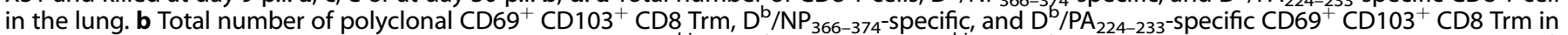

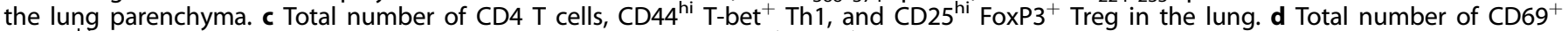
$\mathrm{CD} 11 \mathrm{a}^{\text {h }}$ CD4 Trm in the lung parenchyma. e Total number of CXCR5 ${ }^{+}$PD $-1^{+}$FoxP3 ${ }^{-}$Tfh in the mLN and spleen. Each symbol represents an individual mouse, with bars indicating mean \pm SEM. Statistical analyses performed using Mann-Whitney test. Data from a were pooled from four independent experiments with a total of 13 to 14 mice per group. Data from c, e were pooled from three independent experiments with a total of 6 to 7 mice per group. Data from $\mathbf{b}$, $\mathbf{d}$ were pooled from three independent experiments with a total of 10 to 11 mice per group

\section{DISCUSSION}

The activation of naive $T$ cells is widely thought to require 3 signals, the peptide-MHC complex, the B7 family costimulatory signal, and cytokine. ${ }^{5,6}$ It has long been known that TNFR family members provide additional important signals to sustain the survival of activated T cells, ${ }^{7-12}$ however, the timing of this signal relative to initial priming, the APC type involved and the role of TNFR signaling in secondary lymphoid organs versus the tissues, have not been fully elucidated. Recently, in a chronic infection model with LCMV Clone 13, it was shown that GITR signaling on 
$\mathrm{mLN}$ CD4 T cell, day 3 p.i.

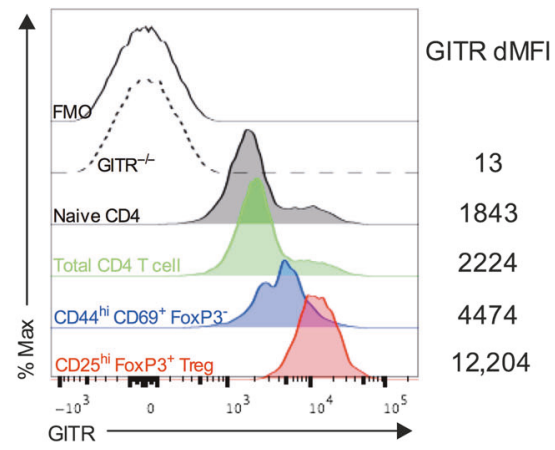

C

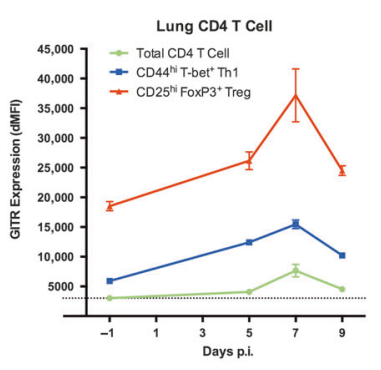

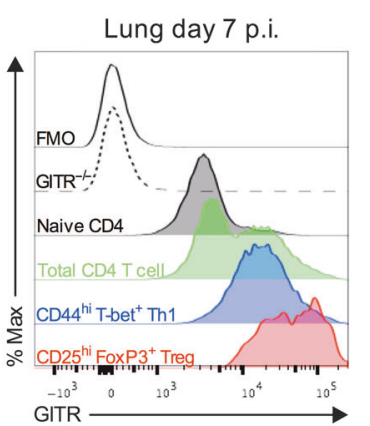

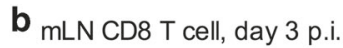

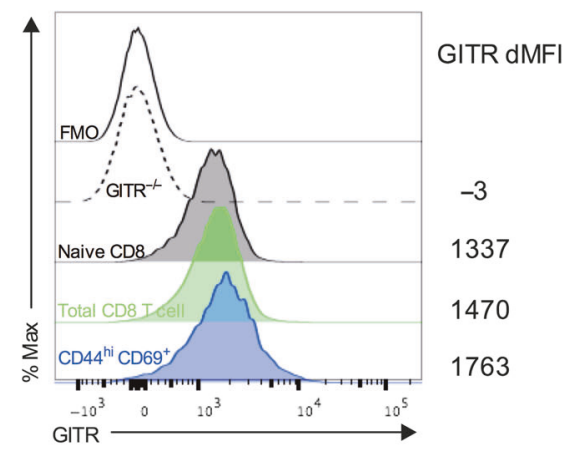

d
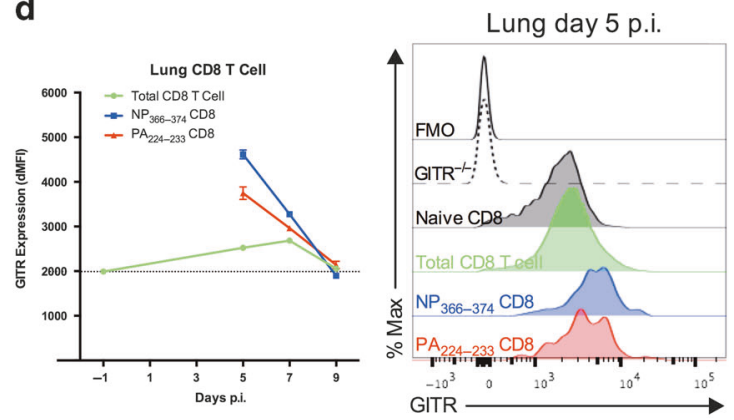

Fig. 6 GITR is upregulated on activated CD4 and CD8 T cells in the $\mathrm{mLN}$ and the lung following influenza infection. Wild-type C57BL/6 mice were infected i.n. with influenza A/HK-X31 and expression of GITR was determined in the $\mathrm{mLN}$ a, $\mathbf{b}$ and the lung c, d. a GITR expression on total CD4 T cell (green), CD44 $4^{\text {hi }} \mathrm{CD} 9^{+}$FoxP3 ${ }^{-}$CD4 T cell (blue), and CD25 hi FoxP3 ${ }^{+}$Treg (red) in the mLN at day 3 p.i. b GITR expression on total CD8 T cell (green), CD44 ${ }^{\text {hi }} \mathrm{CD} 69^{+}$CD8 T cell (blue) in the $\mathrm{mLN}$ at day 3 p.i. c GITR expression kinetics on total CD4 T cell (green), CD44 ${ }^{\text {hi }} \mathrm{T}$ bet $^{+}$Th1 (blue), and CD25 hi FoxP3 ${ }^{+}$Treg (red) in the lung with representative staining at day 7 p.i. shown on the right. $\mathbf{d}$ GITR expression kinetics on total CD8 T cell (green), $\mathrm{D}^{\mathrm{b}} / \mathrm{NP}_{366-374}$-specific (blue), and $\mathrm{D}^{\mathrm{b}} / \mathrm{PA}_{224-233}$-specific CD8 $\mathrm{T}$ cell (red) with representative staining at day 5 p.i. shown on the right. Dashed lines represent baseline/naive expression level of GITR. dMFI refers to the MFI for the specific antibody stain minus the fluorescence minus one (FMO) control. Data points indicate mean \pm SEM. Data from $\mathbf{a}$, $\mathbf{b}$ are representative of two independent experiments, each with three mice. Data from $\mathbf{c}$, $\mathbf{d}$ were pooled from two independent experiments with a total of six mice per time point

CD4 $\mathrm{T}$ cells in the spleen could be temporally and spatially segregated from initial priming, with monocyte-derived inflammatory APCs providing GITRL to primed T cells. Thus, it was proposed that TNF family ligands provide a post-priming signal 4 for T-cell accumulation. ${ }^{7}$ However, the role of signal 4 during an acute infection and its importance in non-lymphoid tissues was unclear. Here we have provided evidence that GITRL on monocyte-derived inflammatory APCs allows increased accumulation of $\mathrm{GITR}^{+/+}$over $\mathrm{GITR}^{-1-}$ T cells in the lung during influenza infection. We also provide evidence that this signal can take place in the $\mathrm{mLN}$ and then again in the lung parenchyma. We further show that GITR costimulation can rescue low-affinity $\mathrm{D}^{\mathrm{b}} / \mathrm{NP}_{366-374^{-}}$ specific CD8 T cells and is critical for the optimal formation of Trm in the lung.

In the context of competition, GITR is required for both CD4 and $\mathrm{CD} 8 \mathrm{~T}$-cell responses to influenza virus. In contrast, in the absence of competition, GITR is required for the CD8 T-cell response, but does not significantly impact the accumulation of the CD4 T cells. A recent study showed that during viral infection in mice, monocyte-derived inflammatory APCs are the main expressers of 4 TNFR superfamily ligands, GITRL, 4-1BBL, OX40L, and CD70, dependent on type I interferon. ${ }^{7}$ In the same study, it was shown that GITR signaling in T cells can regulate molecules involved in adhesion. ${ }^{7}$ Thus, when GITR ${ }^{+/+}$and GITR ${ }^{-1-}$ CD4 T cells compete within the same mouse, it is possible that $\mathrm{GITR}^{+/+} \mathrm{CD} 4 \mathrm{~T}$ cells are more efficient at engaging the inflammatory APCs than their $\mathrm{GITR}^{-/-}$counterparts, thereby having access to survival signals from all 4 TNF family ligands. On the other hand, in the absence of competition, GITR ${ }^{-1-}$ CD4 T cells do not have to compete with their $\mathrm{GITR}^{+/+}$counterparts and are more likely to be able to access the signals from the other TNF family ligands. Thus, other costimulatory molecules such as OX40 and CD27 likely compensate for the lack of GITR on the CD4 T cells in the non-competitive setting. ${ }^{12,34}$ In contrast to the apparent redundancy of GITR with other signals for CD4 T cells, GITR impacts CD8 T cell accumulation even in a non-competitive setting. Similarly, non-redundant roles for accumulation of lung influenza-specific CD8 $T$ cells have also been shown for CD27 and 4-1BB. 8,20,28,35 This greater dependency of CD8 T cell responses on multiple TNFRs compared to CD4 $\mathrm{T}$ cells might reflect the higher demand for these cells to accumulate to resolve a viral infection.

Lung-resident CD4 and CD8 Trm have been shown to play a pivotal role in providing protection against influenza infection, ${ }^{24,25}$ but the factors affecting their establishment in the lung tissue remain incompletely defined. We showed here that the absence of GITR severely impaired the formation of memory $T$ cells in the lung parenchyma as readout at day 30 p.i. As the magnitude of the effect of GITR is already substantial at the effector phase, it appears that the effect of GITR on Trm is in large part due to its impact on the effector precursors. However, further work with conditional deletion of GITR or its ligand will be required to determine whether there is an additional effect of GITR/GITRL on the maintenance of Trm.

We found that GITR ${ }^{+/+}$and GITR ${ }^{-1-}$ OT-Il cells enter the lung equivalently by day 5 , but differences in their accumulation are noted by day 10 . Similarly, a previous study showed that OT-I $T$ cells enter the lung in equal numbers by day 7 post influenza infection with defects in GITR ${ }^{-1-}$ OT-I accumulation detected by day 10 p.i. ${ }^{13}$ Thus, the defect in GITR $^{-1-} \mathrm{T}$ cells does not appear to be due to a failure to traffic to the lung, but rather a 
a Lung, $\times 31$

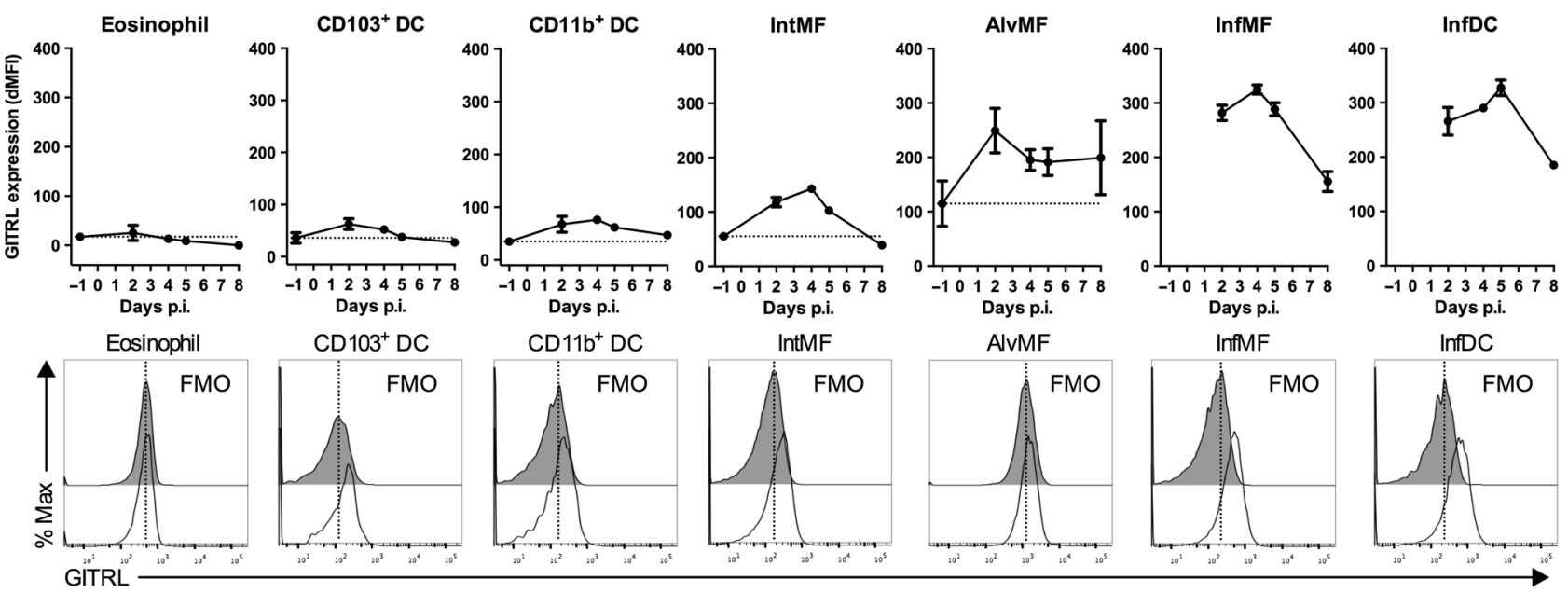

b
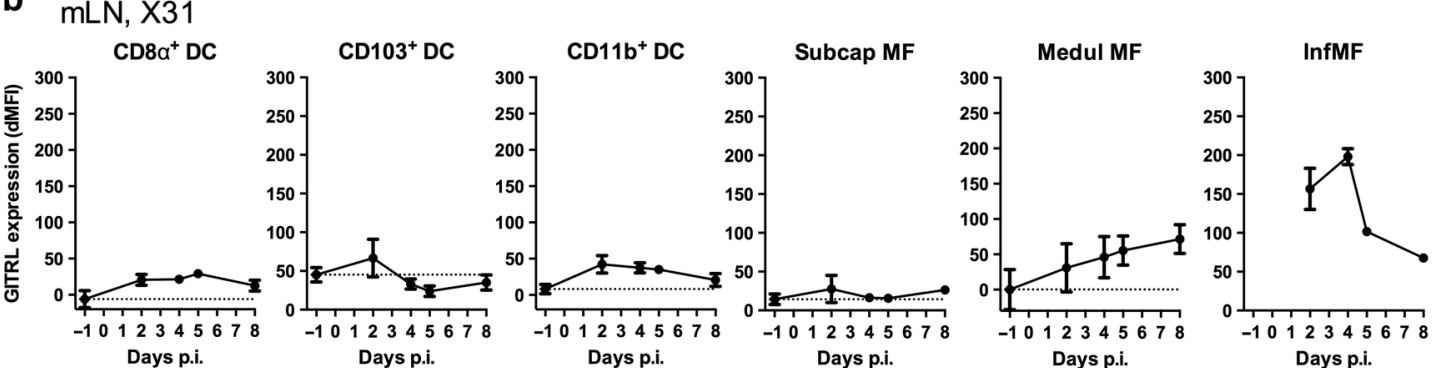

InfDC

$\mathrm{CD} 8 \alpha^{+} \mathrm{DC}$

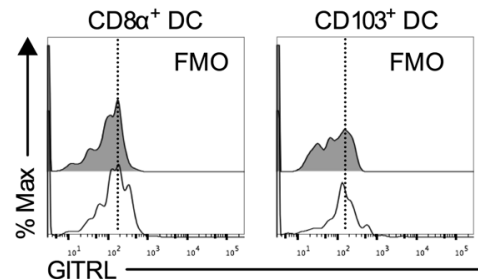

$C D 11 b^{+} D C$
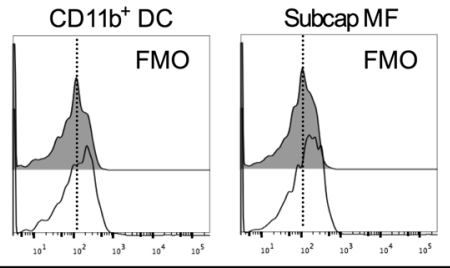

Days p.i.

Days p.i.
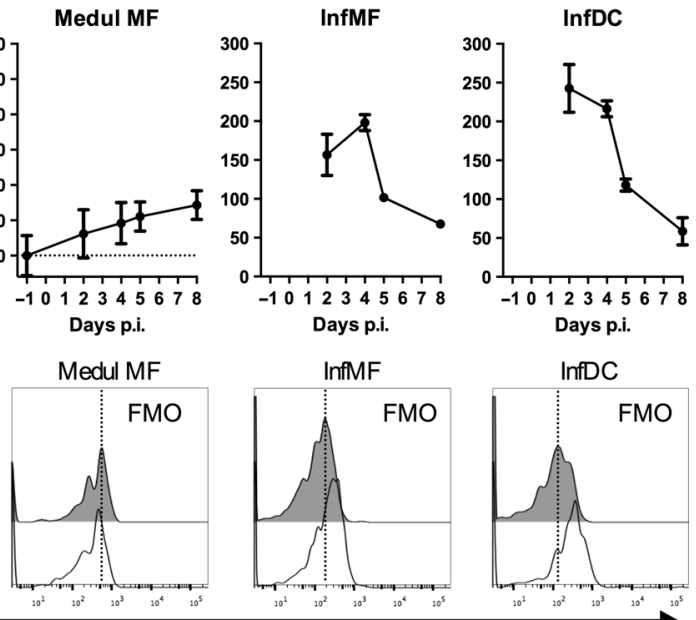

Days p.i.

InfDC

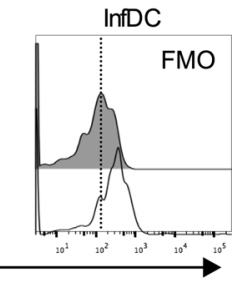

C Lung, $X 31$
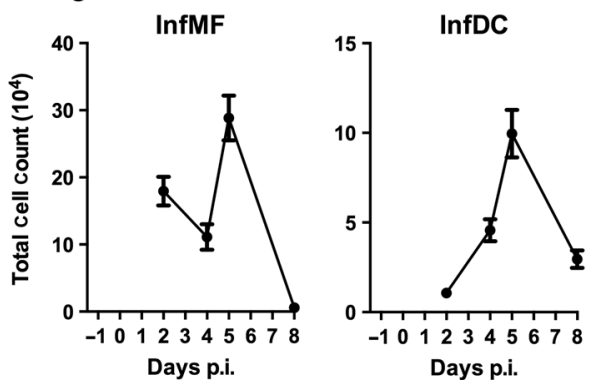

Fig. 7 GITRL expression is highest on monocyte-derived inflammatory APC subsets after influenza X31 infection. Wild-type C57BL/6 mice were infected i.n. with influenza A/HK-X31 and expression kinetics of GITRL determined in the lung and mLN. a Expression kinetics of GITRL on different cell subsets from the lung defined in Supplementary Fig. S2a between day -1 and 8 p.i. with representative staining from day 5 p.i. shown below the kinetics plot. Gray filled histograms represent FMO and open histograms the GITRL staining. $\mathbf{b}$ Expression kinetics of GITRL on different cell subsets from the $\mathrm{mLN}$ defined in Supplementary Fig. S2b between day -1 and 8 p.i. with representative staining shown at day 2 p.i., with GITRL staining shown as open histograms and FMO as gray filled histograms. Horizontal dashed lines in the kinetics plots in a and $\mathbf{b}$ indicate the baseline/naive expression level of GITRL. c Total number of InfMF and InfDC in the lung between day 2 and 8 p.i. dMFI refers to the MFI for the specific antibody stain minus the FMO control. Data points indicate mean \pm SEM. Data in a-c were pooled from two independent experiments with a total of 6-12 mice per time point. IntMF interstitial macrophages; AlvMF alveolar macrophages; InfMF inflammatory macrophages; InfDC inflammatory DCs; Subcap MF subcapsular sinus macrophages; Medul MF medullary macrophages

failure in accumulation or persistence over time. As GITR affects the accumulation/survival rather than the initial rate of division of CD4 and CD8 T cells, ${ }^{13,14}$ the present study supports the hypothesis that GITR provides important survival signals to the effector cells in the lung tissue as they differentiate into Trm.

During the early phase of vesicular stomatitis virus infection, CD8 Trm formation in the small intestine requires signaling 
Lung, day 3 p.i.

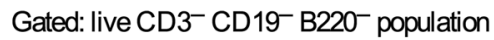

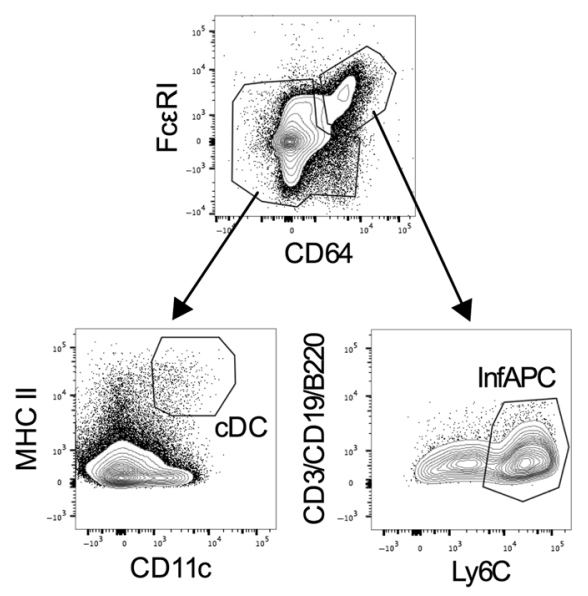

b Lung, day 3 p.i.
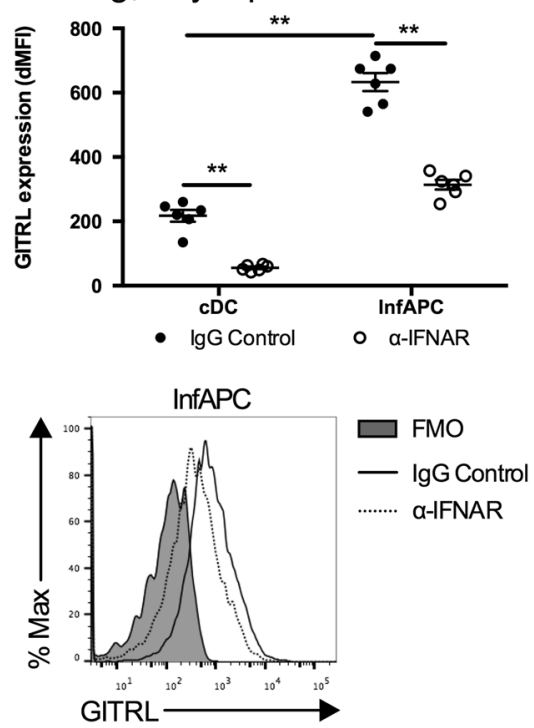

Fig. 8 GITRL expression is mediated in part by IFN-I during influenza infection. a, b Mice were injected i.p. with IFNAR blocking or isotype control antibodies at day -1 and 0 prior to influenza A/PR8 infection. Gating strategy is shown in a and GITRL expression on InfAPC and CDC in the lung at day 3 p.i. summarized in b, with representative staining shown below. dMFI refers to the MFI for the specific antibody stain minus the FMO control. Data from $\mathbf{b}$ pooled from two independent experiments with a total of six mice per group. InfAPC inflammatory APCs

through the mTOR pathway. ${ }^{36}$ The data presented here show that $\mathrm{GITR}^{+1+}$ CD4 and CD8 T cells in the lung parenchyma exhibit higher levels of pS6, a downstream target of mTOR, than their $\mathrm{GITR}^{-1-}$ counterparts. Previous studies have shown a role for GITR in upregulating $\mathrm{pS} 6$ as well as NF-kB, leading to enhanced survival signals, such as $\mathrm{BCl}-\mathrm{X}_{\mathrm{L}}$, and enhanced expression of other TNFRs and cytokine receptors associated with $T$ cell longevity. $7,13,14$

Monocyte-derived inflammatory APCs are the major expressers of GITRL during acute influenza virus infection, as shown here. During chronic LCMV infection there is coordinate induction of GITRL, 4-1BBL, OX40L, and CD70, with preferential expression on inflammatory APC, largely dependent on type I intereferon. ${ }^{7}$ Thus, it is possible that this expression pattern is a general feature of viral infections. A previous study by Ballesteros-Tato et al. suggested that $C D 70$ was mainly expressed by $\mathrm{CD}_{103^{-}} \mathrm{CD} 11 \mathrm{~b}^{\mathrm{hi}}$ DCs during influenza infection. ${ }^{35}$ In that study, FceR1 and/or CD64, markers of monocyte-derived inflammatory APCs, were not used to distinguish CDCs from infAPCs. Thus, it is possible that the $\mathrm{CD}_{103^{-}} \mathrm{CD}_{11 \mathrm{~b}} \mathrm{~b}^{\mathrm{hi}}$ population reported by Ballesteros-Tato et al. also contained the monocyte-derived inflammatory APC population. Recently, using CCR2-deficient mice, Desai et al. showed that inflammatory monocytes were required for persistence of CX3CR1 ${ }^{-}$tissue-resident memory $\mathrm{T}$ cells in the lung after respiratory Vaccinia infection. ${ }^{37}$ These results are consistent with our finding that GITRL on inflammatory monocytes is important for Trm formation.

During primary influenza virus infection, a diverse array of TCRs are used to recognize antigen and there is more or less equivalent expansion of T-cells specific for the $\mathrm{PA}_{224-233}$ and $\mathrm{NP}_{366-374}$ epitopes of influenza virus. However, from primary to secondary influenza infection, the NP response becomes dominant ${ }^{38}$ and this is also accompanied by a narrowing of the TCR repertoire to the $\mathrm{NP}_{366-374}$ epitope with an increased overall TCR affinity. ${ }^{39,40}$ In contrast there is no narrowing of the TCR repertoire to $\mathrm{PA}_{224-233}$ epitope in the memory response. ${ }^{41}$ These results suggest that the TCR repertoire to $\mathrm{NP}_{366-374}$ epitope is more flexible than the TCR repertoire to $\mathrm{PA}_{224-233}$ epitope. Here we found that PA epitopespecific T effector cells are more sensitive to the absence of GITR than the NP-specific effector T cells. The level of tetramer bound per T cell can be used as a surrogate for TCR affinity. ${ }^{21} \mathrm{GITR}^{-1-}$
$\mathrm{NP}_{366-374}$-specific $\mathrm{T}$ cells had on average a $50 \%$ increase in MFI for tetramer binding compared to their GITR ${ }^{+/+}$counterparts within the same mice. Thus, in the absence of GITR, the TCR repertoire to $\mathrm{NP}_{366-374}$ can compensate, by expanding $\mathrm{T}$ cells with higher affinity, whereas there was only a minor change in tetramer MFI for the TCR repertoire to $\mathrm{PA}_{224-233}$. These findings are consistent with previous work suggesting that the PA-specific TCR repertoire is less flexible, and likely explains the greater dependence of PA versus NP epitope-specific T cells on GITR. Interestingly, an increase in TCR affinity to the $\mathrm{NP}_{366-374}$ epitope was also observed in CD27-deficient mice, but whether or not the TCR affinity to $\mathrm{PA}_{224-233}$ epitope is affected by the lack of $\mathrm{CD} 27$ was not examined. $^{21}$

In this study, we used mice expressing a conditional deletion of GITRL exon 2 to test the role of GITRL in rescuing low-affinity influenza-specific CD8 T cells. This mouse was previously shown to express normal levels of GITRL on the cell surface, but with less structural stability, resulting in reduced activity of GITRL, albeit a partial defect compared to mice completely lacking GITR. ${ }^{7}$ Here we found that mice with mutant GITRL in all cells, GITRL ${ }^{\text {exon2 } 2 / \Delta}$ mice, had an $\sim 25 \%$ increase in TCR binding to $\mathrm{NP}_{366-374}$-specific T cells and a similar effect ( $20 \%$ increase), was observed in mice in which exon 2 was deleted only in LysM $^{+}$cells. The relatively similar effect when exon 2 was deleted in all cells as compared to only in $\mathrm{LysM}^{+}$cells, is consistent with the idea that the majority of the GITRL signal comes from monocyte-derived APC rather than from classical DC. The reduced effect in GITRL ${ }^{\text {exon2 } 2 / \Delta}$ mice compared to $\mathrm{GITR}^{-1-}$ mice, is likely due to the residual function of

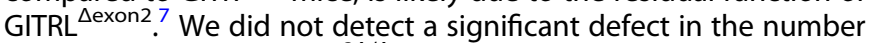
of CD8 T cells in GITRL ${ }^{\text {exon}} 2 \Delta / \Delta$ mice (data not shown), likely due to the fact that GITRL is still expressed on the cell surface with only a minor defect in activity.

There has been growing evidence that T cells, instead of a brief encounter, need repeated encounters with APCs for optimal response against viral infections, including signals from APC in the lungs. ${ }^{42-44}$ However, little is known about the costimulatory requirements for APC interactions in the lung, or whether TNFR family signals are confined to the secondary lymphoid organs. Here, we detected GITR and GITRL early in the mLN as well as in the lung at a time when $T$ cells start to accumulate there. To 
a

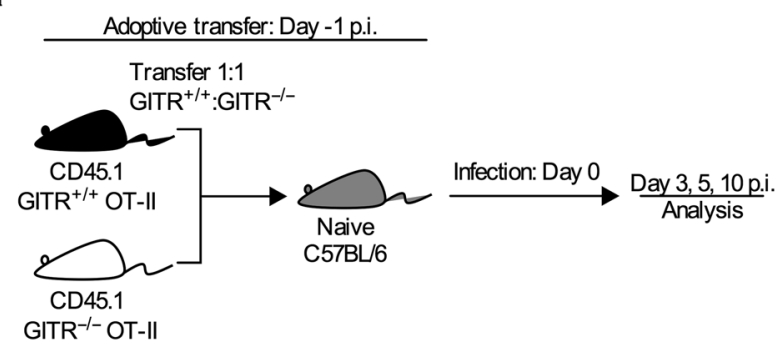

C

$\mathrm{mLN}$, day 3 p.i.

Gated: live CD4 $4^{+}$CD $45.1^{+}$OT-II

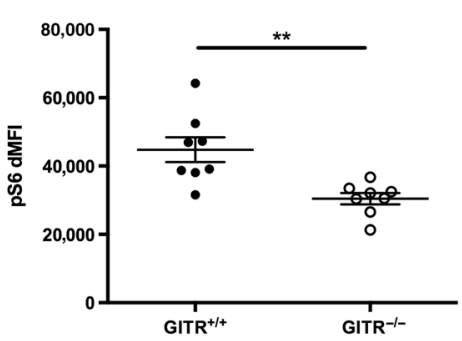

e Day 10 p.i.

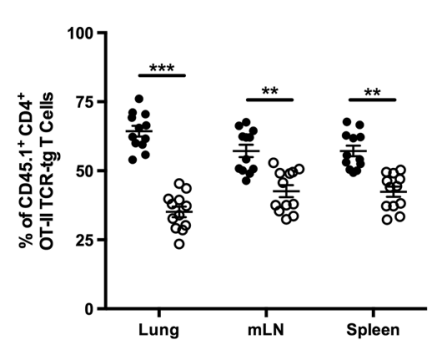

g

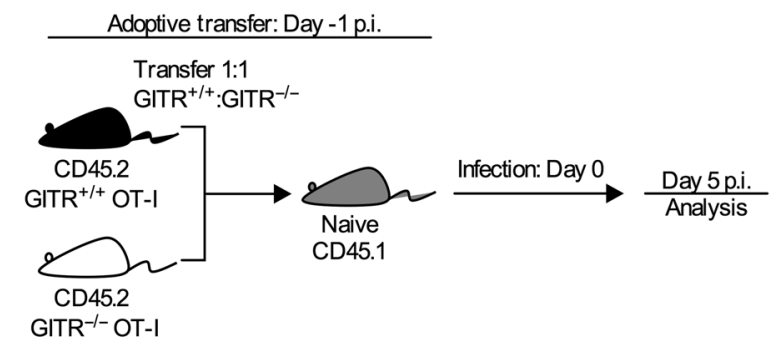

f Day 10 p.i. b

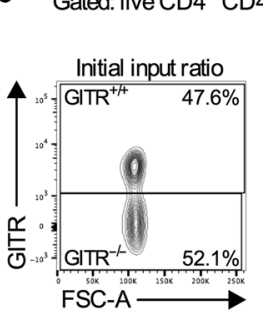

d

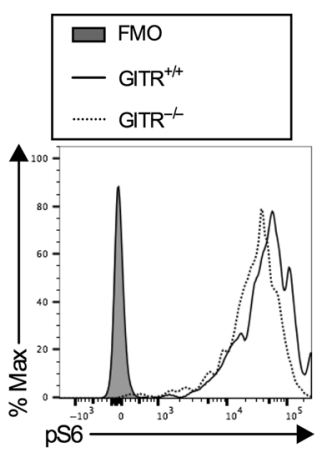

Lung, day 5 p.i.

Gated: live CD4 $4^{+}$CD $45.1^{+}$OT-॥
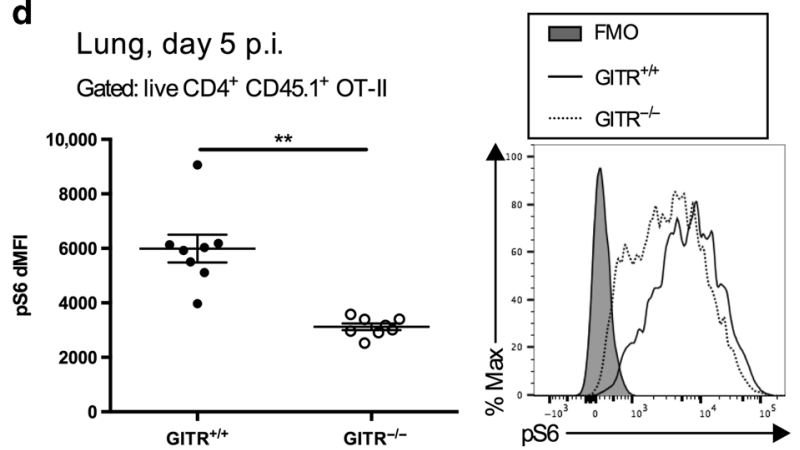
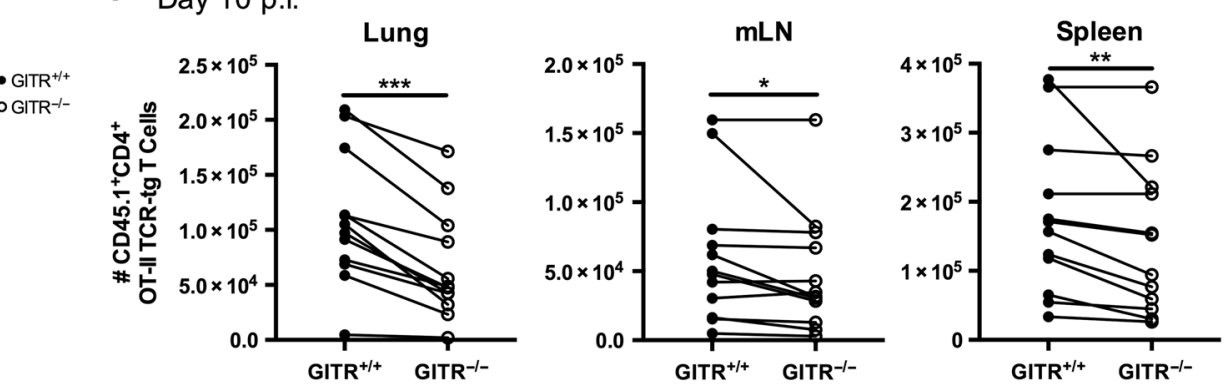

h

Lung, day 5 p.i.

Gated: live CD8 ${ }^{+}$CD $45.2^{+}$OT-I
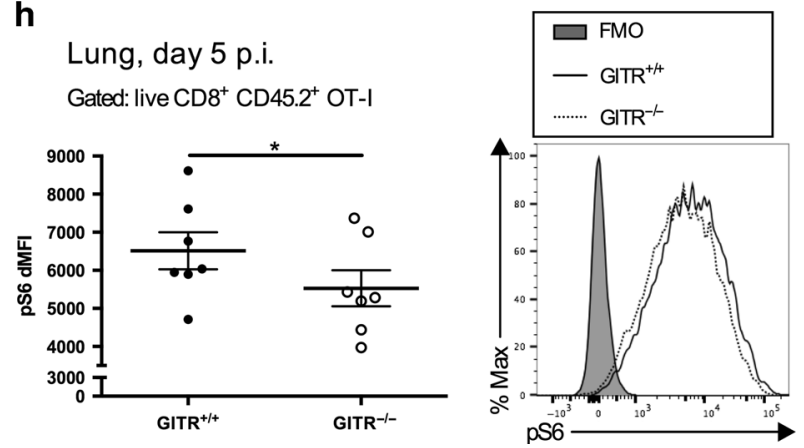

Fig. 9 GITR signaling increases pS6 levels in antigen-specific OT-II CD4 and OT-I CD8 T cells. a Wild-type CD45.2 C57BL/6 mice received a 1:1 mixture of 1 million each $\mathbf{c}$ and $\mathbf{d}$ or 25,000 each $\mathbf{e}$ and $\mathbf{f}$ of GITR $^{+/+}$and GITR ${ }^{-/-}$CD45.1 OT-II isolated from littermates and were infected i.n. the following day with influenza A/PR8-OVAll. b Representative flow plots depicting the initial and the post-infection GITR ${ }^{+/+}:$GITR $^{-/-}$ratio. $\mathbf{c}$ At day 3 p.i., cells isolated from the $\mathrm{mLN}$ were stained directly ex vivo for the level of phosphorylated-ribosomal protein S6 (pS6) with representative staining on the right. d At day 5 p.i., cells isolated from the lung were stained directly ex vivo for the level of pS6 with representative staining on the right. At day 10 p.i., proportions e and total numbers $\mathbf{f}$ of GITR GI+ $^{+/ 1}$ and GITR ${ }^{-/-}$OT-II were determined in the

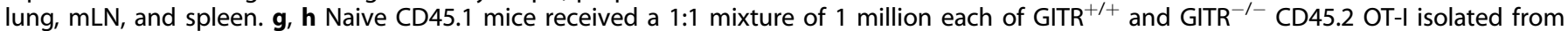
littermates, and were infected i.n. the following day with influenza A/PR8-OVA. $\mathbf{h}$ At day 5 p.i., cells isolated from the lung were stained directly ex vivo for the level of pS6 with representative staining on the right. dMFI refers to the MFI for the specific antibody stain minus the FMO control. Each symbol represents an individual mouse, with bars indicating mean \pm SEM. Statistical analyses performed using Wilcoxon test. Data in c, $\mathbf{d}$ were pooled from two independent experiments with eight mice. Data in $\mathbf{e}$, $\mathbf{f}$ were pooled from two independent experiments with 12 mice. Data in $\mathbf{h}$ were pooled from two independent experiments with seven mice

provide further support that GITR/GITRL is signaling within the $\mathrm{mLN}$ and then in the lung, we compared the level of pS6 in $\mathrm{GITR}^{+/+}$and $\mathrm{GITR}^{-/-}$T cells competing within the same mouse. As phospho-signaling is generally transient, ${ }^{45}$ the finding that $\mathrm{GITR}^{+/+}$
OT-II isolated from mLN and lung had a higher level of pS6 than their GITR $^{-/-}$counterparts argues that GITR/GITRL signaling is occurring first in the $\mathrm{mLN}$ and then again in the lung tissue. Thus, we suggest that signal 4 provides important additional survival 
signals to $\mathrm{T}$ cells in the $\mathrm{mLN}$ as well as when $\mathrm{T}$ cells enter the influenza-infected lung, and that these signals sustain the effectors as well as allowing them to survive the transition to Trm.

\section{METHODS}

Mice

C57BL/6 mice were obtained from Charles River Laboratories (St. Constant, Quebec, Canada). Female mice were used in all experiments except where indicated and were age- and sexmatched within each experiment. GITR ${ }^{-1-}$ mice were previously described $^{14}$ and backcrossed one additional time to C57BL/6 mice or to CD45.1 OT-II mice (kindly provided by David Brooks, Princess Margaret Cancer Center, Toronto, Ontario) or to CD45.2 OT-I (C57BL/6-Tg(TcraTcrb)1100Mjb/J) mice obtained from Jackson Laboratories (Bar Harbor, USA) to generate littermate controls. GITRL $^{\text {exon2fl/fl }}$ mice were previously described. ${ }^{7}$ Briefly, after neomycin resistance gene removal, GITRL ${ }^{\text {exon2fl/fl }}$ mice were bred to homozygosity before crossing to LysM-Cre (B6.129P2-Lyz2 ${ }^{\mathrm{tm} 1}$ (cre)lfo/J) mice to generate LysM-Cre ${ }^{+/+} / \mathrm{LysM}-\mathrm{Cre}^{-/-} \mathrm{GITRL}^{\text {exon2f/ff }}$ littermate controls or to CMV-Cre (B6.C-Tg (CMV-Cre)1Cgn/J) mice to delete GITRL ubiquitously. After confirming germline deletion, the CMV-Cre GITRL ${ }^{\text {exon2 } 2 / \Delta}$ mice were maintained without the Cre transgene (referred to as GITRL ${ }^{\text {exon } 2 \Delta / \Delta}$ mice). For bone marrow chimera experiments, Thy1.1 (B6.PL-Thy $\left.1^{a} / \mathrm{CyJ}\right)$ and CD45.1 (B6. SJL-Ptprc ${ }^{a}$ Pepc ${ }^{b} /$ BoyJ) mice were obtained from Jackson Laboratories (Bar Harbor, USA). All mice were housed under specific pathogen-free conditions in the Division of Comparative Medicine at the Terrence Donnelly Centre for Cellular and Biomolecular Research (University of Toronto). Animal studies were approved by the animal care committee at the University of Toronto in accordance with the Canadian Council on Animal Care.

\section{Reagents and antibodies}

Biotinylated $\mathrm{H}-2 \mathrm{D}^{\mathrm{b}} / \mathrm{NP}_{366-374}$ :ASNENMETM and $\mathrm{H}-2 \mathrm{D}^{\mathrm{b}} / \mathrm{PA}_{224-233}$ : SSLENFRAYV monomers were obtained from the National Institute for Allergy and Infectious Disease tetramer facility (Emory University, Atlanta, GA), and tetramerized with streptavidin APC (ProZyme, San Leandro, CA). All antibodies used for flow cytometry are listed in Supplementary Table S1. Fixable viability dye was purchased from eBioscience (San Diego, CA). Anti-mouse IFNAR1 antibody (Clone: MAR1-5A3) and Mouse IgG1 isotype control were purchased from Bio X Cell (West Lebanon, NH).

\section{Influenza virus infection}

Influenza A/PR8-OVA and A/PR8-OVAll were kindly provided by Paul Thomas and Peter Doherty (St. Jude Children's Research Hospital, Memphis, Tennessee). The tissue culture infectious dose $50\left(\mathrm{TCID}_{50}\right)$ was determined for influenza A/HK-X31, A/PR8, A/PR8OVA, and A/PR8-OVAll viruses by MDCK assays. ${ }^{46}$ Six- to ten-week old mice were anaesthetized with isofluorane and infected intranasally (i.n.) with $30 \mu \mathrm{L}$ of diluted virus at a dose of $5 \mathrm{HAU}$ (equivalent to $3.86 \times 10^{4} \mathrm{TCID}_{50}$ ) of $\mathrm{X} 31$, or $5 \times 10^{5} \mathrm{TCID}_{50}$ of PR8, PR8-OVA, or PR8-OVAll. For PR8, PR8-OVA, or PR8-OVAll infection, mice were monitored daily and killed when moribund.

\section{Tissue harvest and processing}

At the indicated time points after immunization, lung, $m L N$ and spleen were harvested. Lung was perfused with $10 \mathrm{~mL}$ of PBS, and then minced and digested with $2 \mathrm{mg} \mathrm{mL}^{-1}$ of collagenase IV (Invitrogen, Carlsbad, CA) for $45 \mathrm{~min}$ at $37^{\circ} \mathrm{C}$ in a shaker. Lung tissue was mechanically disrupted through a $70 \mu \mathrm{m}$ cell strainer and leukocytes were enriched by isolation over an $80 / 40 \%$ Percoll gradient (GE healthcare, Chicago, IL) after RBC lysis. Spleen (after $\mathrm{RBC}$ lysis) and $\mathrm{mLN}$ were mechanically disrupted through a $70 \mu \mathrm{m}$ cell strainer to generate single cell suspensions. For APC isolation, $\mathrm{mLN}$ was digested with $1 \mathrm{mg} \mathrm{mL}^{-1}$ of collagenase IV for $45 \mathrm{~min}$ at $37^{\circ} \mathrm{C}$ in a shaker. For Trm detection, mice were injected intravenously with $3 \mu \mathrm{g}$ of anti-mouse Thy 1.2 antibody conjugated to FITC and killed 10 min later with tissues processed as described above. Blood was collected from the saphenous vein and treated with RBC lysis buffer.

\section{Flow cytometry}

Freshly isolated single cell suspensions from the lung, $\mathrm{mLN}$, and spleen were treated with Fc Block for $15 \mathrm{~min}$ at $4{ }^{\circ} \mathrm{C}$ followed by surface staining for $30 \mathrm{~min}$ at $4^{\circ} \mathrm{C}$. Staining with $\mathrm{H}-2 \mathrm{D}^{\mathrm{b}}$-restricted tetramers were performed simultaneous with surface staining for $30 \mathrm{~min}$ at $4{ }^{\circ} \mathrm{C}$. Samples were fixed with $4 \%$ paraformaldehyde following surface staining. Intracellular staining, where applicable, was performed for $30 \mathrm{~min}$ at $4{ }^{\circ} \mathrm{C}$ following surface staining described above and permeabilization with FoxP3 Transcription Factor Staining Buffer Set (eBioscience). For intracellular cytokine staining, lung and spleen samples were restimulated ex vivo with $1 \mu \mathrm{M}$ of the $1-A^{d}$-restricted $O A_{323-339}$ peptide or the $H-2 D^{b}$ restricted peptides: $\mathrm{NP}_{366-374}$ or $\mathrm{PA}_{224-233}$ for $6 \mathrm{~h}$ with GolgiStop (BD Biosciences, San Jose, CA) at $37^{\circ} \mathrm{C}$. For endogenous CD4 T-cell cytokine detection, lung and spleen samples were restimulated ex vivo with $500 \mathrm{HAU} / \mathrm{mL}$ of live influenza virus for $18 \mathrm{~h}$ at $37^{\circ} \mathrm{C}$ with GolgiStop added for the final $6 \mathrm{~h}$ of incubation. After restimulation, cells were surfaced stained, fixed and permeabilized (BD Biosciences), and stained for intracellular cytokine production. Unstimulated samples (no peptide) were used as negative controls. Samples were acquired with LSR Fortessa or LSR Fortessa X20 (BD Biosciences) with FACSDiva software and analyzed with Flowjo VX (Tree Star, Inc., Ashland, OR).

T-cell isolation and adoptive transfers

Naive CD4 T cells were purified from spleens of $\mathrm{GITR}^{+/+}$and GITR $^{-/-}$CD45.1 OT-II TCR-tg mice with EasySep Mouse CD4 T-cell Isolation Kit (StemCell Technologies, Vancouver, BC). Naive CD8 T cells were purified from spleens of $\mathrm{GITR}^{+/+}$and $\mathrm{GITR}^{-1-}$ CD45.2 OT-I TCR-tg mice with EasySep Mouse CD8 T Cell Isolation Kit (StemCell Technologies, Vancouver, BC). Samples of either genotype were counted by trypan blue exclusion three times and mixed in a 1:1 ratio. OT-I TCR-Tg CD8 T cell purity, OT-II TCR-Tg CD4 $\mathrm{T}$ cell purity, and $\mathrm{GITR}^{+/+}: \mathrm{GITR}^{-/-}$ratios were confirmed by flow cytometry. Cells from the mixed sample were adoptively transferred intravenously in $200 \mu \mathrm{L}$ volume into recipient mice 1 day prior to infection. For OT-II transfers, $2 \times 10^{6}$ cells were transferred for day 3 and day 5 p.i. analyses, $5 \times 10^{4}$ cells were transferred for day 10 p.i. analyses. For OT-I transfers, $2 \times 10^{6}$ cells were transferred for day 5 p.i. analyses.

\section{Phosphoflow assays}

Lung tissue was processed as described above, except that the digestion with $2 \mathrm{mg} \mathrm{mL}^{-1}$ of collagenase IV was reduced to $30 \mathrm{~min}$ at $37^{\circ} \mathrm{C}$ in a shaker. Phosphoflow staining was performed according to Protocol I described in BD Phosflow Protocols for Mouse Splenocytes. Briefly, following viability dye staining, samples were fixed with BD Phosflow Lyse/Fix Buffer for $11 \mathrm{~min}$ at $37^{\circ} \mathrm{C}$, permeabilized with BD Phosflow Perm/Wash buffer I for 30 min at RT, then stained with antibody mixture for $1 \mathrm{~h}$ at RT.

Mixed bone marrow chimeras

Thy1.1 or CD45.1 host mice were lethally irradiated with two doses of $550 \mathrm{rad}$ and reconstituted with a 1:1 mixture of GITR ${ }^{-1-}$ Thy 1.2 CD45.2:GITR ${ }^{+/+}$Thy1.2 CD45.1 bone marrow cells delivered i.v. for a total of $5 \times 10^{6}$ cells. Reconstituted chimeric mice were given water supplemented with $2 \mathrm{mg} \mathrm{mL}^{-1}$ neomycin sulfate (Bio-Shop, Burlington, ON, Canada) for 2 weeks consecutively, and were rested for a total of 90 days before chimerism in the blood was checked. Chimeric mice were infected according to the indicated schedules. 
In vivo IFNAR1 blockade

For in vivo IFNAR1 blockade, mice were injected i.p. with $500 \mu \mathrm{g}$ of a-IFNAR1 blocking antibody or IgG1 isotype control at day -1 and day 0 (total of $1 \mathrm{mg} / \mathrm{mouse}$ ) prior to influenza infection.

Data analysis and statistics

All statistical analyses were performed using GraphPad Prism 6 (San Diego, CA), with the specific test performed indicated elsewhere. n.s. not significant, ${ }^{*} p<0.05,{ }^{* *} p<0.01,{ }^{* * *} p<0.001$, $* * * * 0<0.0001$ were applied.

\section{ACKNOWLEDGEMENTS}

We thank Ethan Shevach for provision of GITRL ${ }^{\text {exon2f/f/ }}$ mice, David Brooks for provision of CD45.1 OT-II mice, the National Institute of Allergy and Infectious Diseases NIH tetramer core facility for influenza MHC class I monomers, Birinder Ghumman for technical assistance and Jen Gommerman for critical review of the manuscript. This research was funded by Canadian Institutes of Health Research (CIHR) grants MOP-123480 and FDN-143250 to T.H.W., K.C.W., and A.C.Z. were funded by ClHR studentship awards (GSD-134854 to A.C.Z.), and K.-L.C. by an Ontario Graduate Scholarship. T.H.W. holds the Sanofi Pasteur Chair in Human Immunology at the University of Toronto.

\section{AUTHOR CONTRIBUTIONS}

K.-L.C. and T.H.W. designed experiments, analyzed data, and wrote the manuscript. K.L.C., with help from N.V.B., K.C.W., and A.C.Z., performed all experiments.

\section{ADDITIONAL INFORMATION}

The online version of this article (https://doi.org/10.1038/s41385-018-0105-5) contains supplementary material, which is available to authorized users.

Competing interests: The authors declare no competing interests.

\section{REFERENCES}

1. Topham, D. J., Tripp, R. A. \& Doherty, P. C. CD8+ T cells clear influenza virus by perforin or Fas-dependent processes. J. Immunol. 159, 5197-5200 (1997).

2. Brown, D. M., Dilzer, A. M., Meents, D. L. \& Swain, S. L. CD4 T cell-mediated protection from lethal influenza: perforin and antibody-mediated mechanisms give a one-two punch. J. Immunol. 177, 2888-2898 (2006).

3. Moskophidis, D. \& Kioussis, D. Contribution of virus-specific CD8+ cytotoxic T cells to virus clearance or pathologic manifestations of influenza virus infection in a T cell receptor transgenic mouse model. J. Exp. Med. 188, 223-232 (1998).

4. Wissinger, E. L., Saldana, J., Didierlaurent, A. \& Hussell, T. Manipulation of acute inflammatory lung disease. Mucosal Immunol. 1, 265-278 (2008).

5. Schwartz, R. H. Costimulation of T lymphocytes: the role of CD28, CTLA-4, and B7/ BB1 in interleukin-2 production and immunotherapy. Cell 71, 1065-1068 (1992).

6. Curtsinger, J. M. \& Mescher, M. F. Inflammatory cytokines as a third signal for T cell activation. Curr. Opin. Immunol. 22, 333-340 (2010).

7. Chang, Y. H. et al. Dichotomous expression of TNF superfamily ligands on antigen-presenting cells controls post-priming anti-viral CD4(+) T cell immunity. Immunity 47, 943-958 (2017). e949.

8. Lin, G. H. et al. Endogenous 4-1BB ligand plays a critical role in protection from influenza-induced disease. J. Immunol. 182, 934-947 (2009).

9. Chen, L. \& Flies, D. B. Molecular mechanisms of T cell co-stimulation and coinhibition. Nat. Rev. Immunol. 13, 227-242 (2013).

10. Takahashi, C., Mittler, R. S. \& Vella, A. T. Cutting edge: $4-1 B B$ is a bona fide CD8 T cell survival signal. J. Immunol. 162, 5037-5040 (1999).

11. Rogers, P. R., Song, J., Gramaglia, I., Killeen, N. \& Croft, M. OX40 promotes Bcl-xL and $\mathrm{BCl}-2$ expression and is essential for long-term survival of CD4 T cells. Immunity 15, 445-455 (2001).

12. Hendriks, J. et al. CD27 is required for generation and long-term maintenance of T cell immunity. Nat. Immunol. 1, 433-440 (2000).

13. Snell, L. M. et al. CD8 T cell-intrinsic GITR is required for T cell clonal expansion and mouse survival following severe influenza infection. J. Immunol. 185, 7223-7234 (2010)

14. Clouthier, D. L. et al. GITR intrinsically sustains early type 1 and late follicular helper CD4 T cell accumulation to control a chronic viral infection. PLoS Pathog. 11, e1004517 (2015)
15. Clouthier, D. L. \& Watts, T. H. Cell-specific and context-dependent effects of GITR in cancer, autoimmunity, and infection. Cytokine Growth Factor Rev. 25, 91-106 (2014).

16. Shimizu, J., Yamazaki, S., Takahashi, T., Ishida, Y. \& Sakaguchi, S. Stimulation of CD25(+)CD4(+) regulatory $T$ cells through GITR breaks immunological selftolerance. Nat. Immunol. 3, 135-142 (2002).

17. McHugh, R. S. et al. CD4(+)CD25(+) immunoregulatory T cells: gene expression analysis reveals a functional role for the glucocorticoid-induced TNF receptor. Immunity 16, 311-323 (2002).

18. Snell, L. M., Lin, G. H., McPherson, A. J., Moraes, T. J. \& Watts, T. H. T-cell intrinsic effects of GITR and 4-1BB during viral infection and cancer immunotherapy. Immunol. Rev. 244, 197-217 (2011).

19. Nocentini, G. \& Riccardi, C. GITR: a modulator of immune response and inflammation. Adv. Exp. Med. Biol. 647, 156-173 (2009).

20. Ballesteros-Tato, A., Leon, B., Lee, B. O., Lund, F. E. \& Randall, T. D. Epitope-specific regulation of memory programming by differential duration of antigen presentation to influenza-specific CD8(+) T cells. Immunity 41, 127-140 (2014).

21. van Gisbergen, K. P. et al. The costimulatory molecule CD27 maintains clonally diverse CD8(+) T cell responses of low antigen affinity to protect against viral variants. Immunity 35, 97-108 (2011).

22. Nugent, C. T. et al. Characterization of $C D 8+T$ lymphocytes that persist after peripheral tolerance to a self antigen expressed in the pancreas. J. Immunol. 164, 191-200 (2000)

23. Yee, C., Savage, P. A., Lee, P. P., Davis, M. M. \& Greenberg, P. D. Isolation of high avidity melanoma-reactive CTL from heterogeneous populations using peptideMHC tetramers. J. Immunol. 162, 2227-2234 (1999).

24. Wu, T. et al. Lung-resident memory CD8 T cells (TRM) are indispensable for optimal cross-protection against pulmonary virus infection. J. Leukoc. Biol. 95, 215-224 (2014)

25. Turner, D. L. \& Farber, D. L. Mucosal resident memory CD4 T cells in protection and immunopathology. Front. Immunol. 5, 331 (2014).

26. Rosato, P. C., Beura, L. K. \& Masopust, D. Tissue resident memory T cells and viral immunity. Curr. Opin. Virol. 22, 44-50 (2017).

27. Mueller, S. N. \& Mackay, L. K. Tissue-resident memory T cells: local specialists in immune defence. Nat. Rev. Immunol. 16, 79-89 (2016).

28. Zhou, A. C., Wagar, L. E., Wortzman, M. E. \& Watts, T. H. Intrinsic 4-1BB signals are indispensable for the establishment of an influenza-specific tissue-resident memory CD8 T-cell population in the lung. Mucosal Immunol. 10, 1294-1309 (2017).

29. Zens, K. D., Chen, J. K. \& Farber, D. L. Vaccine-generated lung tissue-resident memory $\mathrm{T}$ cells provide heterosubtypic protection to influenza infection. $\mathrm{JCI}$ Insight 1, e85832 (2016).

30. Anderson, K. G. et al. Intravascular staining for discrimination of vascular and tissue leukocytes. Nat. Protoc. 9, 209-222 (2014).

31. Segura, E. \& Amigorena, S. Inflammatory dendritic cells in mice and humans. Trends Immunol. 34, 440-445 (2013).

32. Hammad, H. et al. Inflammatory dendritic cells--not basophils--are necessary and sufficient for induction of Th2 immunity to inhaled house dust mite allergen. J. Exp. Med. 207, 2097-2111 (2010).

33. Ruvinsky, I. et al. Ribosomal protein $\mathrm{S} 6$ phosphorylation is a determinant of cell size and glucose homeostasis. Genes Dev. 19, 2199-2211 (2005).

34. Kopf, M. et al. OX40-deficient mice are defective in Th cell proliferation but are competent in generating $\mathrm{B}$ cell and CTL responses after virus infection. Immunity 11, 699-708 (1999).

35. Ballesteros-Tato, A., Leon, B., Lund, F. E. \& Randall, T. D. Temporal changes in dendritic cell subsets, cross-priming and costimulation via CD70 control CD8(+) T cell responses to influenza. Nat. Immunol. 11, 216-224 (2010).

36. Sowell, R. T., Rogozinska, M., Nelson, C. E., Vezys, V. \& Marzo, A. L. Cutting edge: generation of effector cells that localize to mucosal tissues and form resident memory CD8 T cells is controlled by mTOR. J. Immunol. 193, 2067-2071 (2014).

37. Desai, P., Tahiliani, V., Stanfield, J., Abboud, G. \& Salek-Ardakani, S. Inflammatory monocytes contribute to the persistence of CXCR3(hi) CX3CR1 (lo) circulating and lung-resident memory $\mathrm{CD} 8(+) \mathrm{T}$ cells following respiratory virus infection. Immunol. Cell Biol. 96, 370-378 (2018).

38. Belz, G. T., Xie, W., Altman, J. D. \& Doherty, P. C. A previously unrecognized H-2D (b)-restricted peptide prominent in the primary influenza A virus-specific CD8(+) T-cell response is much less apparent following secondary challenge. J. Virol. 74, 3486-3493 (2000).

39. Zhong, W. \& Reinherz, E. L. In vivo selection of a TCR Vbeta repertoire directed against an immunodominant influenza virus $\mathrm{CTL}$ epitope. Int. Immunol. 16, 1549-1559 (2004).

40. Kedzierska, K., Turner, S. J. \& Doherty, P. C. Conserved T cell receptor usage in primary and recall responses to an immunodominant influenza virus nucleoprotein epitope. Proc. Natl Acad. Sci. USA 101, 4942-4947 (2004). 
GITRL on inflammatory antigen presenting cells in the lung parenchyma... K-L Chu et al.

41. Turner, S. J., Diaz, G., Cross, R. \& Doherty, P. C. Analysis of clonotype distribution and persistence for an influenza virus-specific CD8+ T cell response. Immunity 18, 549-559 (2003).

42. McGill, J., Van Rooijen, N. \& Legge, K. L. Protective influenza-specific CD8 T cell responses require interactions with dendritic cells in the lungs. J. Exp. Med. 205, 1635-1646 (2008).

43. lijima, N., Mattei, L. M. \& Iwasaki, A. Recruited inflammatory monocytes stimulate antiviral Th1 immunity in infected tissue. Proc. Natl Acad. Sci. USA 108, 284-289 (2011).
44. McMaster, S. R. et al. Pulmonary antigen encounter regulates the establishment of tissue-resident CD8 memory $T$ cells in the lung airways and parenchyma. Mucosal Immunol. 11, 1071-1078 (2018).

45. Krutzik, P. O., Irish, J. M., Nolan, G. P. \& Perez, O. D. Analysis of protein phosphorylation and cellular signaling events by flow cytometry: techniques and clinical applications. Clin. Immunol. 110, 206-221 (2004).

46. Cottey, R., Rowe, C. A. \& Bender, B. S. Influenza virus. Curr. Protoc. Immunol. Chapter 19, Unit 1911 (2001). 